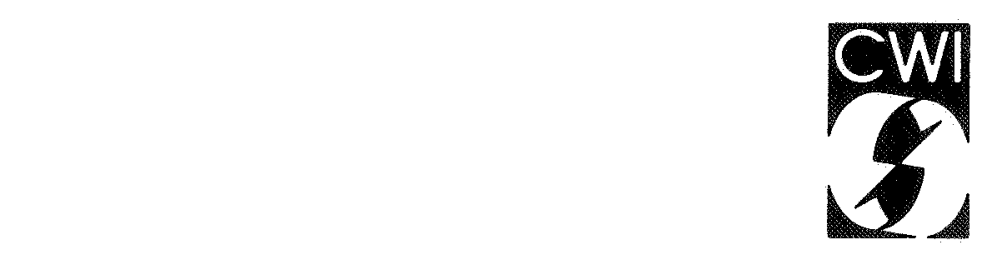

Centrum voor Wiskunde en Informatica Centre for Mathematics and Computer Science

\author{
D.J. Smit
}

String theory and algebraic geometry of moduli spaces 
The Centre for Mathematics and Computer Science is a research institute of the Stichting Mathematisch Centrum, which was founded on February 11, 1946, as a nonprofit institution aiming at the promotion of mathematics, computer science, and their applications. It is sponsored by the Dutch Government through the Netherlands Organization for the Advancement of Pure Research (Z.W.O.).

$32 G 15,81920$

Copyright @ Stichting Mathematisch Centrum, Amsterdam 


\title{
String Theory and Algebraic Geometry of Moduli Spaces
}

\author{
Dirk-Jan Smit \\ Institute for Theoretical Physics \\ Princetonplein 5, P.O. Box 80.006 \\ 3508 TA Utrecht, The Netherlands
}

\begin{abstract}
It is shown how the algebraic geometry of the moduli space of Riemann surfaces entirely determines the partition function of Polyakov's string theory. This is done by using elements of Arakelov's intersection theory applied to determinants of families of differential operators parametrized by moduli space. As a result we write the partition function in terms of a exponentials of Arakelov's Green functions and Faltings' invariant on Riemann surfaces. Generalizing to arithmetic surfaces, i.e. surfaces which are associated to an algebraic number field $K$, we establish a connection between string theory and the infinite primes of $K$. As a result we conjecture that the usual partition function is a special case of a new partition function on the moduli space defined over $K$.
\end{abstract}

\section{INTRODUCTION AND SUMMARY}

Recently it has become clear that Polyakov's formulation for quantizing a string theory [38] has a profound geometrical interpretation. In this formulation one considers the string partition function $Z$, expressed as a perturbation series over random surfaces together with an integration over the space of metrics $M_{p}$ of a Riemann surface $M$ of genus $p$, and an integration over the space $\mathcal{E}$, containing all embeddings of the surface into $d$-dimensional space-time:

$$
\begin{aligned}
& x: M \rightarrow \mathbb{R}^{d} \quad x \in \mathcal{E} \\
& Z=\sum_{p=0}^{\infty} \int_{M_{p} \times \mathcal{E}} d g d x \exp (-S[x, g]), g \in M_{p} .
\end{aligned}
$$

We assume that both the two-dimensional worldsheet swept out by the string, as well as the $d$ dimensional space-time in which the string moves can be Wick-rotated to Euclidean spaces. Furthermore, we shall restrict ourselves to closed strings so that we will be dealing exclusively with closed Riemann surfaces.

The action $S[x, g]$ in (1.2) is Polyakov's action for the bosonic string, which is quadratic in $x$ [38]

$$
S[x, g]=\frac{1}{2} \int_{M} \sqrt{g} g^{a b} \partial_{a} x \partial_{b} x .
$$

We consider (1.2) for a given genus $p>1$, so the object of interest is

$$
Z_{p}=\int_{M_{p} \times \varepsilon} d g d x \exp -S[x, g] .
$$

The aim of this paper is to show that, after gauge fixing of the symmetries of $S[x, g]$, the integrand in (1.4) defines a natural holomorphic volume form on moduli space. Futhermore, when Polyakov's prescription is generalized to include arithmetic surfaces, we show, using the arithmetic geometry set up by FALTINGS [20], that the partition function of a string theory is essentially determined by the infinite primes of an algebraic number field.

We begin by summarizing the gauge fixing procedure which reduces the infinite dimensional integral over $M_{p}$ in (1.4) to a finite dimensional one over moduli space. From the physical point of view one requires (1.4) to be a single valued function. So it is natural to require that the volume form in (1.4) must have the same symmetries as the classical action (1.3). To construct such a volume form

Report PM-R8705

Centre for Mathematics and Computer Science

P.O. Box 4079, 1009 AB Amsterdam, The Netherlands 
we make use of the fact that the tangent space $T_{g} M_{p}$ at $g \in M_{p}$, is algebraically reducible

$$
T_{g} M_{p}=\stackrel{\circ}{T}_{p} \oplus \hat{T}_{g} M_{p}
$$

where $\stackrel{\circ}{T} M_{p}$ contains the $g$-trace, while $\hat{T}_{g} M_{p}$ is spanned by the traceless deformations of the metric. Correspondingly the measure $d g$ in (1.4) factorizes formally as

$$
d g=d \tau d h, d \tau \in \stackrel{\circ}{T} M_{p}, d h \in \hat{T}_{g} M_{p} .
$$

The classical action is invariant under the group of diffeomorphisms Diff $(M)$ and the conformal group $\operatorname{Conf}(M)$. On $M_{p}$, the action of an element $\phi$ of $\operatorname{Diff}(M)$ is given by the Lie derivative $\nabla$ of $g$ w.r.t. the vector field $V$, the infinitesimal parameter of $\phi$ in Diff $(M)$, while Conf $(M)$ acts through the conformal factor $\sigma: g \rightarrow e^{\sigma} g$, where $g$ is an element of $M_{p}$ and $\sigma(z)$ is a scalar function. The combined action of $\operatorname{Conf}(M) \times \operatorname{Diff}_{o}(M)$ on $M_{p}$ is given by

$$
\delta g_{a b}=\delta \sigma g_{a b}+g_{a b}(\nabla \cdot V)+(P V)_{a b}
$$

where $\operatorname{Diff}_{o}(M)$ is the identity component of $\operatorname{Diff}(M)$, i.e. it consists of the diffeomorphisms continuously connected to the identity, and the operator $P$ projects into the traceless part of $T_{g} M_{p}$ :

$$
(P V)_{a b}=\nabla_{a} V_{b}+\nabla_{b} V_{a}-g_{a b}(\nabla \cdot V) \text {. }
$$

It is well known that the measure in $Z_{p}$ cannot be invariant under both $\operatorname{Diff}(M)$ and $\operatorname{Conf}(M)$. We choose it to be invariant under $\operatorname{Diff}(M)$, so a physical state in the theory will be an equivalence class $(x, g)$ w.r.t. the action of Diff $(M)$. To isolate the action of Diff $(M)$ one applies the Faddeev-Popov procedure. Locally the metric $g$ is pure gauge, i.e. all metrics are locally equivalent. However, on a generic Riemann surface with $p>0$ this is not globally the case: there are deformations of the metric which cannot be obtained through diffeomorphisms and conformal transformations. These deformations are the Teichmüller deformations of the metric. Together with the traceless diffeomorphic variations, they span $\hat{T}_{g} M_{p}$, hence it is seen that the Teichmüller deformations belong to the kernel of the adjoint of $P, \operatorname{Ker} P^{\dagger}$. (For $p>1, \operatorname{Ker} P$ is a trivial vector space.)

Teichmüller space $\sigma_{p}$ is obtained by factoring out $\operatorname{Conf}(M) \times D$ iffo $(M)$ from $M_{p}$,

$$
\sigma_{p} \simeq M_{p} /\left(\operatorname{Conf}(M) \times \operatorname{Diff}_{o}(M)\right)
$$

which is the space of conformal equivalence classes of Riemann surfaces of genus $p$. Its dimension is given by the dimension of Ker $P^{f}$ which is $6 p-6$. Choosing real coordinates $t_{1}, \ldots, t_{6 p-6}$ on $\mathfrak{T}_{p}$ one can rewrite the integration over $M_{p}$, by a change of variables, as a successive integration over $\operatorname{Conf}(M), \operatorname{Diff}(M)$ and $\mathscr{T}_{p}$. We will drop the integration over Diff $(M)$. Then (1.4) can be written as $[17,23,34]$ :

$Z_{p}=\int_{C o n f(M)} d \sigma \int_{\sigma_{p}} d t_{1} \wedge \cdots \wedge d t_{6 p-6}\left(\frac{\operatorname{det}^{\prime}\left(P^{\dagger} P\right)}{\operatorname{det}\left(S_{i}, S_{j}\right)}\right)^{\frac{1}{2}}\left(\frac{\operatorname{det}^{\prime} \Delta}{\int_{M} \sqrt{g}}\right)^{-d / 2}$

where $\left\{S_{i}\right\}_{i=1}^{6 p-6}$ forms a basis for Ker $P^{\dagger}$ and all determinants are regularized by means of the $\zeta$ function regularization, where we omit the zero eigenvalues, c.f. [12].

On Teichmüller space we still have the action of the so-called mapping class group $\Gamma_{p}=\operatorname{Diff}(M) / \operatorname{Diff}_{o}(M)$, a discrete group, whose elements are represented (mod $\operatorname{Diff}_{o}(M)$ ) by diffeomorphisms that are not continuously deformable to the identity. The integrand in (1.10) is not invariant under the action of $\Gamma_{p}$, which implies that if we factor out $\Gamma_{p}$ from $\mathcal{G}_{p}$, we get an obstruction due to these global diffeomorphisms. In other words, the integral in (1.10) does not define a function on the space

$$
\mathfrak{R}=\mathfrak{T}_{p} / \Gamma_{p}
$$

The space $\mathfrak{T}$ is called the moduli space of Riemann surfaces of genus $p$. The action of $\Gamma_{p}$ on $\mathscr{T}_{p}$ is 
not free, so $\mathscr{K}$ is in fact a $V$-manifold or orbifold [5]. Because the classical action (1.3) is invariant under all diffeomorphisms it is natural to require that we should construct a partition function having the same invariance, that is, the integration over $\sigma_{p}$ should be replaced by an integration over $\Re$.

This amounts to studying a possible obstruction of the global diffeomorphisms. Such an obstruction means that there are no suitable global coordinates on $\mathfrak{T}$. We will show however by using the complex algebraic geometry of moduli space, that one can construct a globally defined invariant volume form out of (1.10), which can be integrated over $\Re$.

The paper is organized as follows. In section 2, we will present some mathematical background material which is used to rewrite the partition function as a section of a determinant line bundle. The construction of an invariant volume form then reduces to giving a Hermitean metric on this bundle. For this purpose we make use of certain elements of Faltings' work on Arakelov geometry. In section 3 we then show how this material combines with the results of QUILLEN [39] together with the socalled Grothendieck-Riemann-Roch theorem which leads to the conclusion that the Hermitean metric on the determinant line bundle describing the integral in $Z_{p}$ is holomorphically flat in the critical dimension. We thus recover the Belavin-Kniznik theorem [7] on moduli space, which states that if the theory is conformally invariant, the integrand of the partition function is the square modulus of a holomorphic function. We will write this function in terms of Faltings' invariant and exponentials of Arakelov Green's functions.

Finally, in section 4 we generalize Polyakov's formulation to arithmetic surfaces, associated with an algebraic number field $K$. We then exhibit how the partition function of a string theory is given in terms of the contribution of the infinite primes associated with $K$ to the so-called modular height of the $K$-rational points in $T$. This leads to the conjecure that the integral over moduli space in (1.10) can be considered as a special case of a partition function involving arithmetic surfaces, which is a summation over discrete objects.

\section{DETERMINANT LINE BUNDLES AND THE STRUCTURE OF THE PARTITION FUNCTION}

In this section we begin our analysis of the determinants that appear in the string partition function (1.10) using complex geometry. First we will present some basic properties of complex line bundles on a Riemann surface in terms of sheaf cohomology. This material will be used throughout this paper. (A rigorous treatment of sheaf cohomology can be found in [26]). Subsequently we will explain in 2.2 which line bundles are of relevance in string theories. This leads to the observation that the real determinants in (1.10) can be rewritten in terms of complex determinants of Laplacians of CauchyRiemann operators.

In 2.3 we will introduce a determinant bundle as a generalization of the usual determinant of an elliptic operator to an operator which has a non-zero index and which acts on an infinite dimensional vectorspace. The problem of defining an integrable volume element in (1.10) then amounts to putting a suitable Hermitean metric on such a bundle. As a first step in this construction, we will, by introducing certain aspects of Arakelov's intersection theory, discuss a theorem of Faltings. Using this theorem one can compute a volume form on the formal difference $H^{0}(M, L)-H^{l}(M, L)$, where $L$ is a Hermitean admissible line bundle on a Riemann surface $M$. One thus obtains a metric on each fibre of the determinant bundle.

\subsection{Complex line bundles and divisors on a Riemann surface}

A line bundle $L$ over $M$ can be constructed in terms of open coverings $\left\{U_{\alpha}\right\}$ of $M$ together with a set of local trivializations $\phi_{\alpha}:\left.L\right|_{u_{\alpha}} \rightarrow U_{\alpha} \times \mathbb{C}$. The transition function $g_{\alpha \beta}=\phi_{\alpha}{ }^{\circ} \dot{\phi}_{\beta}{ }^{-1}$, satisfying the cocycle condition, is a section over $U_{\alpha} \cap U_{\beta}$ of $\theta\left(U_{\alpha} \cap U_{\beta}\right)$, the sheaf of holomorphic functions. One could equally well consider other trivilizations by taking $f_{\alpha} \in \mathcal{O}^{*}\left(U_{\alpha}\right)$ which is the sheaf of nowhere zero holomorphic functions on $U_{\alpha}$, and define $f_{\alpha}^{\prime}=f_{\alpha} \phi_{\alpha}$ with $h_{\alpha \beta}=\left(f_{\alpha} / f_{\beta}\right) g_{\alpha \beta}$ as transition function.

Assigning a set of local trivializations to every covering $U_{\alpha}$ gives rise to the space of holomorphic 
sections of $\theta^{*}$, which is denoted by $H^{0}\left(M, \vartheta^{*}\right)$; similarly the space of sections of a line bundle $L$ is given by $H^{0}(M, L)$. The 1-cocycles $g_{\alpha \beta} \in \mathcal{O}^{*}\left(U_{\alpha} \cap U_{\beta}\right)$ of a line bundle $L$ define elements of a group, the first cohomology group $H^{1}\left(M, \Theta^{*}\right)$. Actually $H^{1}\left(M, O^{*}\right)$ is the group of isomorphism classes of line bundles over $M$. So, an element of $H^{1}\left(M, \theta^{*}\right)$ determines a line bundle up to isomorphisms. We can get more information from $H^{1}\left(M, \theta^{*}\right)$, in particular information on the existence of globat trivializations by considering the exact sheaf sequence:

$$
0 \rightarrow \underset{\mathbb{Z}}{i} \rightarrow \exp ^{\rightarrow} \theta^{*} \rightarrow 0
$$

where $\mathbb{Z}$ is the constant sheaf, i.e. $\mathbb{Z}\left(U_{\alpha}\right)=\mathbb{Z}$ for all $U_{\alpha}, i$ is the inclusion map of $\mathbb{Z}$ into $\theta$ and exp the exponential map; i.e. if $f \in \theta\left(U_{\alpha}\right)$ then $\exp (f)=e^{2 \pi i f}$. The kernel of $\exp (f)$ is precisely the image of $\mathbb{Z}$ in $\mathcal{O}$ hence (2.1.1) is indeed exact. For any exact sequence like (2.1.1) we have a long exact sequence for cohomology groups, which for (2.1.1) reads

$$
\begin{aligned}
& 0 \rightarrow H^{0}(M, \mathbb{Z}) \rightarrow H^{0}(M, \mathcal{\theta}) \rightarrow H^{0}\left(M, \theta^{*}\right) \rightarrow \\
& \rightarrow H^{1}(m, \mathbb{Z}) \rightarrow H^{1}(M, \mathcal{\theta}) \rightarrow H^{1}\left(M, \theta^{*}\right) \rightarrow \\
& \rightarrow H^{2}(M, \mathbb{Z}) \rightarrow H^{2}(M, \mathcal{\theta}) \rightarrow \cdots
\end{aligned}
$$

The $H^{0}$ part in (2.1.2) is just the exact sequence of Abelian groups:

$$
0 \rightarrow \mathbb{Z} \rightarrow \mathbb{C} \rightarrow \mathbb{C}^{*} \rightarrow 0
$$

which is induced by $f$. (This is because the global holomorphic functions on $M$ are necessarily constant). Furthermore, since every compact Riemann surface is, as a topological space, homeomorphic to a sphere with $p$ handles, we have $H^{2}(M, \mathbb{Z})=\mathbb{Z}$ and $H^{1}(M, \mathbb{Z})=\mathbb{Z}^{2 p}$, while $H^{1}(M, \mathcal{O}) \simeq \mathbb{C}^{p}$. Thus, from (2.1.2) we deduce the following exact sequence

$$
0 \rightarrow \mathbb{C}^{P} / \mathbb{Z}^{2 p} \rightarrow H^{1}\left(M, \theta^{*}\right) \rightarrow \mathbb{Z} \rightarrow 0 .
$$

Therefore we can assign an integer, to any line bundle $L$ over $M$, called the degree of $L$. If we have a metric on the line bundle, one can express the degree of $L$ as

$$
\operatorname{deg}(L)=\int_{M} c_{1}(L)
$$

where $c_{1}(L)$ is called the first Chern class of $L$, represented by the curvature form of the metric on $L$. We now conclude from (2.1.3) that $L$ is completely characterized by its first Chern class and an element in $H^{1}(M, \theta) / H^{1}(M, \mathbb{Z}) \simeq \mathbb{C}^{p} / \mathbb{Z}^{2 p}$ the group of holomorphic flat line bundles over $M$. This is a complex torus, and in fact it is an Abelian variety, called the Picard variety or the Jacobian variety, of $M$. The first Chern class can be interpreted as the obstruction to a global trivialization of $L$. Namely, if $c_{1}(L)=0$, then the local functions representing an element of $H^{1}(M, \mathcal{O})$ corresponding to the isomorphism class of $L$ in $H^{1}\left(M, \theta^{\star}\right)$ define a global trivialization.

It is useful to have a description of line bundles in terms of divisors. A divisor $D$ on $M$ is a formal finite sum of points on $M: D=\sum_{i} n_{i} P_{i}$ where $n_{i}$ are integers, $P_{i} \in M$. The divisors on $M$ form a group, denoted by $\operatorname{Div}(M)$. The connection with line bundles can be established as follows. Suppose we restrict $D \in \operatorname{Div}(M)$ to an open domain $U_{\alpha} \subset M$. One can always find a meromorphic function $f_{\alpha}$ of which the zeros and poles coincide with $D$ restricted to $U_{\alpha}:\left.D\right|_{u_{\alpha}}=\Sigma n_{i} P_{i}, P_{i} \in U_{\alpha}$ and we can construct the transition function $g_{\alpha \beta}=f_{\alpha} / f_{\beta} \in \mathcal{O}^{*}\left(U_{\alpha} \cap U_{\beta}\right)$. The line bundle given by the transition functions $\left\{g_{\alpha \beta}=f_{\alpha} / f_{\beta}\right\}$ is called the associated line bundle, denoted by $[D]$. Choosing a different function $f_{\alpha}^{\prime}$ to define $D$, then $f_{\alpha} / f_{\alpha}^{\prime} \in \mathcal{O}^{*}\left(U_{\alpha}\right)$ and $g_{\alpha \beta}^{\prime}=f_{\alpha}^{\prime} / f_{\beta}=g_{\alpha \beta}\left(f_{\alpha}^{\prime} / f_{\alpha}\right)\left(f_{\beta}^{\prime} / f_{\beta}\right)^{-1}$. This function defines an isomorphic line bundle. A consequence of this is that if $D$ and $D^{\prime}$ are two 
divisors, given by $\left\{f_{\alpha}\right\}$ and $\left\{f_{\alpha}^{\prime}\right\}$, then $D+D^{\prime}$ is given by $\left\{f_{\alpha} f_{\alpha}^{\prime}\right\}$, hence

$$
\begin{aligned}
& {[D+D]=[D] \otimes\left[D^{\prime}\right]} \\
& {[D]^{\star}=[-D] .}
\end{aligned}
$$

Now suppose that $D$ is the divisor of a meromorphic function: $D=(f)$, then the transition functions are the identity, 1 , implying that $[D]$ is the trivial line bundle. This amounts to define an equivalence relation between the divisors as follows. We say that $D$ and $D^{\prime}$ are linearly equivalent, written as $D \sim D^{\prime}$ if $D=D^{\prime}+(f)$, or equivalently, if $[D]=\left[D^{\prime}\right]$. It is thus seen that [] is actually a map from the group of divisors to the group of isomorphism classes of line bundles modulo linear equivalence. This group is called the Picard $\operatorname{group} \operatorname{Pic}(M)$

$$
\text { [ ] } \operatorname{Div}(M) \rightarrow \operatorname{Pic}(M) \simeq H^{1}\left(M, \theta^{*}\right) \text {. }
$$

Properties of holomorphic and meromorphic sections of line bundles are conveniently discussed in terms of divisors. We associate to a divisor $D$ the space $\mathcal{L}(D)$ of meromorphic functions on $M$ for which $D+(f) \geqslant 0$, i.e. these functions are holomorphic on $M-\bigcup_{i} P_{i}$, with $\operatorname{Ord}_{p_{i}}(f) \geqslant-n_{i}$, where $\operatorname{Ord}_{p_{i}}(f)$ is the order of the zero or the pole of $f$ at a point $P_{i}$. Now let $s_{0}$ be a global meromorphic section of $[D]$ with divisor $D=\left(s_{0}\right)$. Then for any global holomorphic section $s$ of $[D]$, the quotient $s / s_{0}$ is a meromorphic function on $M$ with

$$
\begin{aligned}
& \left(s / s_{0}\right)=(s)-\left(s_{0}\right) \geqslant D, \text { i.e. } \\
& s / s_{0} \in \mathscr{L}(D), \text { and }(s)=D+\left(s / s_{0}\right) .
\end{aligned}
$$

On the other hand if $f \in \mathfrak{E}(D)$, then the section $s=f s_{0}$ of the line bundle $[D]$ is holomorphic, hence we conclude that multiplication by $s_{0}$ gives an identification with the space of holomorphic sections of $[D]:$

$$
\mathscr{C}(D) \stackrel{s_{0}}{\rightarrow} H^{0}(M, \mathcal{O}[D])
$$

More precisely: let $D=\Sigma n_{i} P_{i}$ a positive divisor on $M$ and $s_{0} \in H^{0}(M, \theta[D])$ a section of $[D]$ with divisor $D$. Then tensoring with $s_{0}$ gives an identification between the meromorphic functions on $M$ with poles of order $\leqslant n_{i}$ at $P_{i}$ and the holomorphic sections of $[D]$.

We now introduce some notation. For a line bundle $L, L(D)$ denotes the sheaf of meromorphic sections of $L$ with poles of order $\leqslant n_{i}$ at $P_{i}$ and $L(-D)$, the sheaf of sections of with zeors of order $\geqslant n_{i}$ at $P_{i}$. It is thus seen that the identification (2.1.6) is made by multiplication with either $s_{0}$ or its inverse $s_{0}^{-1}$ :

$$
\begin{aligned}
& L(D) \simeq \theta(L \otimes[D]) \\
& L(-D) \simeq \theta(L \otimes[-D]) .
\end{aligned}
$$

Later on, the following exact sheaf sequence plays an important role:

$$
0 \rightarrow(L \otimes Q[-P]) \stackrel{s_{0}}{\rightarrow} Q(L) \rightarrow L[P] \rightarrow 0
$$

where $L[P]$ is the fibre of $L$ at $P$. More precisely, it is the skyscraper sheaf concentrated at $P$ with fibre $L_{P}$ at $P$. From this we deduce the following exact sequence for cohomology groups

$$
\begin{aligned}
& 0 \rightarrow H^{0}(M, L(-P)) \rightarrow H^{0}(M, L) \rightarrow H^{0}(M, L[P]) \rightarrow \\
& \rightarrow H^{1}(M, L(-P)) \rightarrow H^{1}(M, L) \rightarrow 0 .
\end{aligned}
$$

Where we used the identification (2.1.7). (The sequence terminates since $L[P]$ is concentrated at a point).

What we now have seen is that a divisor gives a line bundle with a section, namely the function 
representing it has its poles and zeros at the points specified by the divisor. In this context there is a special section, called the distinguished or unit section. It is constructed through a divisor consisting of a single point $P$. On a neighbourhood $U$ of $P$ one defines the divisor $D=P$ by the function $f(z)=z$, where $z$ is a local coordinate on $U$, such that $f(P)=0$. Introducing $V=M-\{P\}$ one defines the transition function $g_{U V}=z$ on $U \cap V$. The unit section $1_{Q(P)}$ can now be defined in the notation as above by putting $s_{0}=1$ and $s=g_{U V} s_{0}$. Then $1_{O(P)}$ takes the value 1 on $U$ and has a first order zero at $z=P$. It extends meromorphically in an obvious way to all of $M$. Likewise, one can define $\mathbf{1}_{(-P)}$ which has a first order pole at $z=P$. Furthermore one can define for any divisor $D$ a unit section $1_{Q(D)}$ by taking tensor products.

\subsection{Cauchy-Riemann operators}

Let us now use the formalism in 2.1 to describe the operators that appear in the string partition function. We consider the space $T(n)$ of smooth sections of $K^{\otimes n}$, where $K$ is the canonical line bundle an $M$ (i.e. $K=\mathbb{C}\{d z\}$, where $z$ is local complex coordinate). The space $T(n)$ is formed by tensors $T=T(z)(d z)^{n}$, transforming under a change of coordinates $z \rightarrow w(z)$ as $T(w)=\left(\frac{d w}{d z}\right)^{n} T(z)$. The power $n$ can be integer or half integer. Following the notation of $[2,3,17]$ we define covariant derivatives for the elements in $T(n)$ :

$$
\begin{aligned}
& \nabla_{n}^{z}: T(n) \rightarrow T(n+1) ; \quad \nabla_{n}^{z} T=g^{z \bar{z}} \partial_{\bar{z}} T \\
& \nabla_{z}^{n}: T(n) \rightarrow T(n-1) ; \quad \nabla_{z}^{n} T=\left(g_{z \bar{z}}\right)^{n} \partial_{\bar{z}}\left(\left(g^{z \bar{z}}\right)^{n} T\right)
\end{aligned}
$$

where $g_{z \bar{z}}$ is the local metric on $M$ which can be brought into the form: $g_{z \bar{z}} d z d \bar{z}=e^{\sigma} d z d \bar{z}$. (We denote by $g^{z \bar{z}}$ the inverse of $g_{z \bar{z}}$ ).

The operators $\nabla_{n}^{z}$ are the usual Cauchy-Riemann operators, that is, the kernel of $\nabla_{n}^{z}$ is formed by the holomorphic sections of $K^{\otimes n}$, the holomorphic $n$-differentials $(d z)^{n}$. The line bundle of these differentials is denoted by $\Omega^{\otimes n}$, hence we have

$$
\operatorname{Ker} \nabla_{n}^{z} \simeq H^{0}\left(M, \Omega^{\otimes n}\right)
$$

Using the operators $\nabla_{n}^{z}$ and $\nabla_{n}^{z}$, one constructs Laplacians

$$
\begin{aligned}
& \Delta_{n}^{+}: T(n) \rightarrow T(n): \Delta_{n}^{+}=\nabla_{z}^{n+1} \nabla_{n}^{z} \\
& \Delta_{n}^{-}: T(n) \rightarrow T(n): \Delta_{n}^{-}=\nabla_{n-1}^{z} \nabla_{z}^{n} .
\end{aligned}
$$

The spectrum of non zero eigenvalues of $\Delta_{n}^{+}$and $\Delta_{n}^{-}$are identical. To deal with the zero modes we need a duality property of cohomology groups and the Riemann-Roch-theorem [29]. For any line bundle $L$ over $M$, the following duality holds

$$
H^{1}(M, L) \simeq H^{0}\left(M, \Omega \otimes L^{-1}\right)^{\star}
$$

which is known as Serre duality [29]. (The asterisk denotes the dual space). The Riemann-Rochtheorem states

$$
\operatorname{dim} H^{0}(M, L)-\operatorname{dim} H^{1}(M, L)=\operatorname{deg} L-p+1
$$

which, using (2.2.5), is written as

$$
\operatorname{dim} H^{0}(M, L)-\operatorname{dim} H^{0}\left(M, \Omega \otimes L^{-1}\right)=\operatorname{deg} L-p+1 .
$$

Suppose $L$ is a trivial line bundle, then $\operatorname{dim} H^{0}(M, L)=1$ since there is only one global holomorphic function on $M$, i.e. the constant function. From (2.2.7) we thus have

$$
\operatorname{dim} H^{0}(M, \Omega)=p .
$$

For $L=\Omega$ we find, using the above result and (2.2.7) that 


$$
\operatorname{deg} \Omega=2 p-2
$$

Using these results we can compute the index of $\nabla_{n}^{z}$ :

$$
\begin{aligned}
\text { Ind } & \nabla_{n}^{z} \equiv \operatorname{dimKer} \nabla_{n}^{2}-\operatorname{dimCoker} \nabla_{n}^{z} \\
& =\operatorname{dim} H^{0}\left(M, \Omega^{\otimes n}\right)-\operatorname{dim} H^{1}\left(M, \Omega^{\otimes n}\right) \\
& =\operatorname{dim} H^{0}\left(M, \Omega^{\otimes n}\right)-\operatorname{dim} H^{0}\left(M, \Omega^{\otimes(1-n)}\right) \\
& =n(\operatorname{deg}(\Omega))-p+1=(2 n-1)(p-1) .
\end{aligned}
$$

Furthermore, we can apply the Kodaira vanishing theorem [29] which says that if for a line bundle $L$ $\operatorname{deg} L<0$ then $\operatorname{dim}^{0}(M, L)=0$, so that in fact $\operatorname{dim} \operatorname{Coker} \nabla_{n}^{z}=0$ if $n>1$, and therefore

$$
\operatorname{dim} \operatorname{Ker} \nabla_{n}^{z}=(2 n-1)(p-1), n>1 \text {. }
$$

As an application of this formalism, we rewrite the real determinants in the partition function (1.10) in terms of the Laplacians (2.2.4). We observe that the real determinant det $\Delta$ of the Laplacian $\Delta$ in (1.10) is identified with $\left|\operatorname{det} \Delta_{1}\right|$. Writing the action of $P$ in (1.8) in complex coordinates:

$$
P\left(V^{z} \partial_{z}+c . c .\right)=g_{z \bar{z}} \partial_{\bar{z}} V^{\bar{z}} d z d z+c . c \text {. }
$$

it follows that (restricting to the holomorphic part)

$$
\operatorname{Ker} P=H^{0}\left(M, \Omega^{-1}\right)=\operatorname{Ker} \nabla_{z}^{-1}
$$

This is the space of holomorphic vector fields on $M$. It follows from above that for genus $p>1$ $\operatorname{dim} \operatorname{Ker} \nabla_{z}^{-1}=0$. On the other hand we have

$$
\text { Coker } P=\operatorname{Ker} P^{\dagger}=H^{1}\left(M, \Omega^{-1}\right) \simeq H^{0}\left(M, \Omega^{2}\right)=\operatorname{Ker} \nabla_{2}^{z}
$$

which is the space of holomorphic quadratic differentials, which is, according the Riemann-Roch theorem, of complex dimension $3 p-3$. Thus, the space of quadratic differentials is identified with the cotangent space of moduli space. Subsequently, since $P^{\dagger} P$ splits up into two parts, we have: $\left(\operatorname{det} P^{\dagger} P\right)^{\frac{1}{2}}=\left|\operatorname{det} \Delta_{2}\right|$. This reformulation of determinants of real Laplacians into determinants of complex Laplacians $\Delta_{n}$ is useful, because they can be expressed, as we shall see, in terms of their holomorphic square root det $\nabla_{n}^{z}$ which has nice properties.

\subsection{Metrical properties of determinants of Cauchy-Riemann operators}

Because the index of $\nabla_{n}^{2}$ is non zero, and because it acts on an infinite dimensional vector space, it is impossible to define the determinant of $\nabla_{n}^{z}$ in the usual way. In this subsection a generalization of the definition of a determinant is discussed, following the results presented in $[11,22,39]$. Subsequently we introduce the Faltings metric which associates a volume with such a generalization.

Let $V$ be a finite dimensional vector space, and consider a linear map $\delta: V \rightarrow V$. The determinant of $V$ can be defined by taking the highest exterior power $\wedge^{\max } V$, which is called the determinant map det $\delta$. An explicit representation follows if we introduce basisvectors $\left\{V_{i}\right\}_{i=1}^{n}$ on $V$, since

$$
\operatorname{det} \delta\left(V_{1} \wedge \cdots \wedge V_{n}\right)=\delta V_{1} \wedge \cdots \wedge \delta V_{n}
$$

which is just multiplication by a complex number. This is a map $\wedge^{\max } V \rightarrow \wedge^{\max } V$, or equivalently, an element of $\left(\wedge^{\max } V\right)^{\star} \otimes \wedge^{\max } V$

Now we generalize this to the case where we have a linear elliptic operator $D$ acting between two different vector spaces $V_{1}$ and $V_{2}$ of the same dimension

$$
D: V_{1} \rightarrow V_{2} \text {. }
$$

Taking exterior powers we have the induced map 


$$
\operatorname{det} D: \wedge^{\max } V_{1} \rightarrow \wedge^{\max } V_{2}
$$

The determinant $\operatorname{det} D$ is now defined to be an element of the 1-dimensional linear space.

$$
\operatorname{det} D \in\left(\wedge^{\max } V_{1}\right)^{\star} \otimes\left(\wedge^{\max } V_{2}\right)
$$

An extension to the case where $V_{1}$ and $V_{2}$ are infinite dimensional can be made when $D$ is a Fredholm operator of index zero. For such an extension, we first recall the exact sequence

$$
0 \rightarrow \operatorname{Ker} D \rightarrow V_{1} \stackrel{D}{\rightarrow} V_{2} \rightarrow \text { Coker } D \rightarrow 0
$$

from which it follows, using the fact that ker $D$ and Coker $D$ are both finite dimensional vector spaces, that the definition

$$
\operatorname{det} D=\left(\wedge^{\max } \operatorname{Ker} D\right)^{*} \otimes\left(\wedge^{\max } \operatorname{Coker} D\right)
$$

makes sense in this case.

If the operators $D$ have a non zero index we do the following. Consider a family of operators $D_{y}$, varying smoothly with a complex parameter $y \in Y$, where $Y$ is a complex manifold. For each $y \in Y$ (2.3.6) defines a complex 1-dimensional linear space. This fits together yielding a section det $D$ of a line bundle $\mathscr{L}_{y}$ over the parameter space $Y$, called the determinant line bundle

$$
e_{y} \rightarrow Y: e_{y}=\left(\wedge^{\max } \operatorname{Ker} D_{y}\right)^{\star} \otimes\left(\wedge^{\max } \text { Coker } D_{y}\right) \text {. }
$$

This definition of the determinant line bundle makes sense also when the index of $D_{y}$ is non-zero [39]. However the section det $D$ is only nontrivial if $D$ is invertible, i.e. if the index of $D$ vanishes. If the index is nonvanishing det $D \equiv 0$ by definition. So the only way to define the determinant of the Cauchy-Riemann operator $\nabla_{n}^{z}$ is as a section of a determinant line bundle. We postpone the construction of this bundle until the next section. For the moment we focus our attention to properties of the 1-dimensional complex space

$$
\left(\wedge^{\max } H^{0}\left(M, \Omega^{\otimes n}\right)\right)^{\star} \otimes \wedge^{\max }\left(H^{\mathrm{l}}\left(M, \Omega^{\otimes n}\right)\right) .
$$

We will denote this space in the sequel by $\operatorname{det} R \Gamma\left(M, \Omega^{\otimes n}\right)^{-1}$. Using Serre duality and the Kodaira vanishing theorem, it follows that for $n>1$, det $R \Gamma\left(M, \Omega^{\otimes n}\right)^{-1}$ is given by

$$
\left(\operatorname{det} R \Gamma\left(M, \Omega^{\otimes n}\right)\right)^{-1}=\left(\wedge^{\max } H^{0}\left(M, \Omega^{\otimes n}\right)\right)^{*}
$$

and for $n=1$

$$
(\operatorname{det} R \Gamma(M, \Omega))^{-1}=\left(\wedge^{p} H^{0}(M, \Omega)\right)^{\star} \otimes H^{0}(M, \mathcal{O})
$$

(For $n=0$ we have the dual of $(2.3 .9 \mathrm{~b})$ ). Note that by the argument given below $(2.10), H^{0}(M, \mathcal{O})$ is trivial.

Putting a metric on (2.3.9) is equivalent with defining a volume form on the difference $H^{0}\left(M, \Omega^{\otimes n}\right)-H^{1}\left(M, \Omega^{\otimes n}\right)$. This can be viewed as a generalization of the usual case (2.3.1) where det $\delta$ defines a volume for $V$. There exists a natural metric on det $R \Gamma\left(M, \Omega^{\otimes n}\right)$ which turns out to be crucial in the construction of an integrable volume form a moduli space. We discuss it in some detail.

One first constructs a metric on the Riemann surface which induces a natural metric, the so called Arakelov metric [4], on the bundles $\Omega^{\otimes n}$. To construct it we choose an orthonormal basis $\left(w_{1}, \ldots, w_{p}\right)$ in $H^{0}(M, \Omega)$. On $H^{0}(M, \Omega)$ we have the natural Hermitean metric given by the inner product:

$$
\left\langle w_{1}, w_{2}\right\rangle=\frac{i}{2} \int_{M} w_{1} \wedge \bar{w}_{2}
$$

We will fix a metric $g$ on the Riemann surface by requiring that it corresponds to the Kähler form

$$
\omega=\frac{i}{2 p} \sum_{j=1}^{p} w_{j} \wedge \bar{w}_{j}
$$


We will use this metric on the Riemann surface throughout this paper, in particular the covariant derivatives in (2.2.1-2) have been computed with this metric.

Now, suppose we have given a line bundle $L$ together with a Hermitean inner product on the fibre of $L$ of each point $z \in M$. These inner products vary smoothly with $z$. Denote by $\|\cdot\|$ the induced norm on the fibres of $L$, and let $s$ any section of $L$. The first Chern class $c_{1}(L)$ satisfying (2.11) is given by:

$$
c_{1}(L)=\frac{\partial^{2}}{\partial z \partial \bar{z}} \log \|s\|^{2} d z \wedge d \bar{z} \quad s \in L .
$$

The line bundle $L$ over $M$ is called admissible w.r.t. the metric (2.3.10) if

$$
c_{1}(L)=2 \pi i \operatorname{deg}(L) \omega .
$$

This metric is also reffered to as the Arakelov metric on $L$. Arakelov proved [4] that in fact each line bundle over $M$ has an admissible metric w.r.t. (2.3.10) which is unique up to scalar multiplication.

Associated with this metric one can define in the following way, a Green's function. Let $P$ and $Q$ be two given points on $M$ and let $z$ a complex coordinate on $M$. Then there exists a real valued, non negative function $G(P, z)$ of $z$ satisfying the following properties:

a) For $z=P$ it has a first order zero

b) $\frac{\partial^{2}}{\partial z \partial \bar{z}} \log G(P, z) d z \wedge d \bar{z}=2 \pi i \omega$

where $\omega$ is the volume form (2.3.11);

c) $\int_{M} \log G(P, z) \omega=0$

d) $\stackrel{M}{G}(P, Q)=G(Q, P)$; i.e. $G$ is a symmetric function.

One can show easily that if $\Delta$ is the complex scalar Laplacian acting on a function $f$ on $M$, then for $P, Q \in M$

$$
f(Q)=\int_{M} \log G(P, Q) \Delta f(Q) \omega .
$$

The function $\log G(P, Q)$ has a logarithmic singularity at $Q=P$ :

$$
\log G(P, Q)=\log |z|+\mathbb{C}^{\infty} \text {-function. }
$$

One calls the function $g(P, Q) \equiv \log G(P, Q)$ the Green's function of $M$ associated with the metric $g$.

Using the function $G(P, Q)$, one can explicitly construct an admissible metric on $\Omega$ as follows. Consider $\mathcal{O}_{M}(Q)$, the sheaf of holomorphic functions at $Q \in M$, and the unit section $1_{Q(P)}$ at the point $P$. One puts a unique Hermitean metric \|\| on $\Theta_{M}(Q)$ by letting

$$
\|\mathbf{1}\|_{\mathcal{Q}(P)}=G(P, Q)=\exp (g(P, Q))
$$

where $\|\mathbb{1}\|_{Q(P)}$ is the norm of the unit section $\mathbb{1}_{Q(P)}, P \in M$. It is obvious that this is an admissible metric. By tensoring we obtain an admissible metric on $O(D)$ for each divisor $D$. This metric is called the Green's metric on $O(D)$.

Now we do the following. Let $P$ be a point on $M$, and let $\mathcal{O}(P)$ have its Green's metric. Furthermore, suppose we have a differential $f d z$, which has a pole of order one at $z=P$. ( $z$ is a local coordinate at $P$.) Then the residue of this differential gives an isomorphism from the fibre at $P$ of the line bundle

$$
\Omega(P)=\Omega \otimes O(P)
$$

to $\mathbb{C}$. One can do this for all points $P$, such that, if $\mathbb{C}$ has its canonical metric $\|$, the residue map is an isometry. To see this explicitly, consider the generating section at $P$, which is $f d z$. Then the norm of this section at $P$ is given by:

$$
\|f d z\|(P)=\left|r e s_{P} f d z\right|
$$


where the residue is given by

$$
r e s_{p} f d z=\lim _{z \rightarrow P} \frac{f d z}{z-P} .
$$

Using the fact that the residue map is an isometry we thus see that we may put a norm on $\Omega$ by defining:

$$
\|\Omega\|=\left|\operatorname{res}_{P} f d z\right| \lim _{z \rightarrow P} \frac{|z-P|}{G(z, P)} .
$$

By taking tensorial powers, we thus obtain an admissible metric on $\Omega^{\otimes n}$. the reason we use this metric on $\Omega^{\otimes n}$ is because it is possible to "transfer" this metric onto the space det $R \Gamma\left(M, \Omega^{n}\right)^{-1}$. The metric on det $R \Gamma\left(M, \Omega^{\otimes n}\right)$ is referred to as the Faltings metric. To construct it one needs the following theorem.

ThEOREM 1 (Faltings [20]). There exists for any line bundle $L$ over $M$ with an admissible metric on $L$ a unique way to assign a Hermitean metric on the space

$$
\text { det } R \Gamma(M, L) \equiv \wedge^{\max } H^{0}(M, L) \otimes\left(\wedge^{\max } H^{1}(M, L)\right)^{*}
$$

such that the following properties hold

1) An isometry $f: L \rightarrow L^{\prime}$ induces an isometry from $\operatorname{det} R \Gamma(M, L)$ to $\operatorname{det} R \Gamma\left(M, L^{\prime}\right)$.

2) If the metric on $L$ is changed by a factor $\alpha>0$ then the metric on $\operatorname{det} R \Gamma(M, L)$ is changed by $\alpha^{\chi(L)}$ where

$$
\begin{aligned}
\chi(L) & =\operatorname{dim} H^{0}(M, L)-\operatorname{dim} H^{1}(M, L) \\
& =\operatorname{deg} L-p+1
\end{aligned}
$$

3) The metrics on det $R \Gamma(M, L)$ are compatible with the Green's metrics on $Q(D)[P]$ in the following sense. Suppose $D_{1}$ and $D$ are divisors on $M$ such that $D=D_{1}+P, P \in M$. Then the isomorphism

$$
\text { det } R \Gamma(M,(D)) \simeq \operatorname{det} R \Gamma\left(M,\left(D_{1}\right)\right) \otimes \vartheta(D)[P],
$$

which is induced by the exact sequence (c.f. 2.1.8a-b)

$$
0 \rightarrow \Theta\left(D_{1}\right) \rightarrow \Theta(D) \rightarrow \Theta(D)[P] \rightarrow 0,
$$

is in fact an isometry.

4) The metric on det $R \Gamma(M, \Omega)=\wedge^{p} H^{0}(M, \Omega)$ is the one determined by the canonical scalar product on $H^{0}(M, \Omega)$.

We will give the line of reasoning in the proof of this theorem, because it has an interesting consequence in string theory. It is essentially enough to prove property 1, because the Faltings metric may be put on the det $R \Gamma(M, L)$ 's in a unique way so that properties $2,3,4$ hold. Recall that one can construct always a divisor $D$ such that $\theta(D)$ and $\Omega$ are isomorphic as line bundles. Since we have the Green's metric on $O(D)$, which is a scalar multiple of the fixed metric on $\Omega$, conditions 2 and 4 determine the metric on det $R \Gamma(M, O(D))$. With property 3 it then follows that one may determine a metric on det $R \Gamma\left(M, O\left(D^{\prime}\right)\right)$, for any divisor $D^{\prime}$ by adding or subtracting points. Since $G(P, Q)$ is symmetric in $P, Q$ the order in which one adds or subtracts points is irrelevant for the metric on $\operatorname{det} R \Gamma\left(M, O\left(D^{\prime}\right)\right)$.

So it remains to prove that for any isometry $\theta(D) \simeq Q\left(D^{\prime}\right)$ induces an isometry

$$
\operatorname{det} R \Gamma(M, \mathcal{O}(D)) \simeq \operatorname{det} R \Gamma\left(M, \mathscr{O}\left(D^{\prime}\right)\right) \text {. }
$$

For this purpose one adds or subtracts points such that the divisors $D$ and $D^{\prime}$ are both of degree $p-1$. Then they can be written as

$$
E-\sum_{i=1}^{r} P_{i}
$$


for a fixed divisior $E$ and some points $P_{1}, \ldots, P_{r}$ on $M$. For $\mathscr{P}=\left(P_{1}, \ldots, P_{r}\right) \in M^{r}$, $\left(M^{r}=M \underset{1}{\otimes} \cdots \otimes \otimes_{r}\right)$, define $L(\mathscr{P})$ to be $\Theta\left(E-\sum_{i=1}^{r} P^{r}\right)$. One then proceeds in proving property 1) by constructing a line bundle $N$ on $M^{r}$ whose fibre at $\mathscr{P}$ is naturally identified with $\operatorname{det} R \Gamma(M, L(\mathscr{P}))$. The isomorphism classes of this line bundle are easily obtained since they belong to the Picard group of line bundles of degree $p-1, P i c_{p-1}(M)$. We thus have a mapping

$$
\psi: M^{r} \rightarrow P i c_{p-1}(M)
$$

which sends $\left(P_{1}, \ldots, P_{r}\right)$ to $\left(E-\sum_{i=1}^{r} P_{i}\right)$.

Now, recall that in $\operatorname{Pic}_{p-1}(M)$ there is the theta divisor $\theta$ consisting of trivial bundles over $M$. It is proved (e.g. explicitly in [35]) that the bundle $N$ is in fact given by the pull back of $\psi$ :

$$
N=\psi^{*} \theta(-\theta)
$$

in which the unit section of $\theta(-\theta)$ goes over in the meromorphic section $s$ of $N$. Since the fibre of $N$ at $P$ has been identified with det $R \Gamma(M, L(\mathscr{P}))$, the metrics that have been put on $\operatorname{det} R \Gamma(M, L)$ yield a metric \|\|$_{N}$ on $N$. Property 1 ) is thus proved if the curvature of this metric is equal to the curvature of the Hermitean metric $\|\cdot\|_{\theta}$ on $\theta(-\theta)$. This can be done by using the Green's metric on $\theta(D)$. (We refer to [20] for this part of the proof.)

Important for us is, that appearantly there exists a scalar $\Delta$ such that

$$
\|\cdot\|_{N}=\Delta\|\cdot\|_{\theta} .
$$

When appropriately normalized, this factor can be expressed in terms of the new invariant $\delta(M)$ on a Riemann surface introduced in, ref. 20 (pp.401-403). To describe it we will compute the Faltings metric on det $R \Gamma(M, L)$ explicitly.

For the reader's convenience we first explain how property 3 of theorem 1 works. We restrict to the case where $L$ is of degree $d \geqslant 2 p-1$, so that $\operatorname{dimH}(M, L)=0$ by the Kodaira vanishing theorem. Similar as above let $\left\{P_{i}\right\}_{i=1}^{r}$ be $r$ distinct points on $M$, such that $r=d+1-p=\operatorname{dim} H^{0}(M, L)$, and

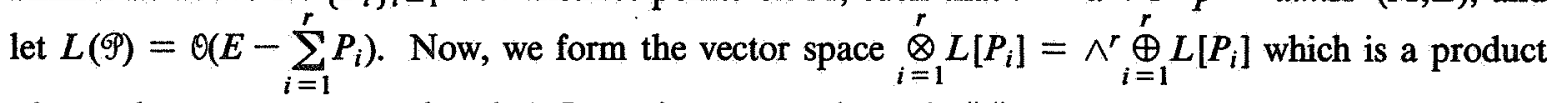
of complex vector spaces of rank 1 . It carries a natural metric $\|\cdot\|_{\text {nat }}$ coming from each component in $\bigoplus_{i=1}^{r} L\left[P_{i}\right]:$

$$
\|\cdot\|_{\text {nat }}=\left\|\alpha_{1} \otimes \cdots \otimes \alpha_{r}\right\|_{\text {nat }}=\Pi_{i}\left\|a_{i}\right\| .
$$

On the space $\underset{i}{\otimes} L\left[P_{i}\right]$ one defines the Faltings metric $\|\cdot\|_{F}$ as

$$
\|\cdot\|_{F}=\|\cdot\|_{n a t} \prod_{i<j} \frac{1}{G\left(p_{i}, p_{j}\right)} .
$$

Using the exact sequence

$$
0 \rightarrow L\left(-\sum_{i=1}^{r} P_{i}\right) \rightarrow L \rightarrow \bigoplus_{i=1}^{r} L\left[P_{i}\right] \rightarrow 0
$$

one obtains the isomorphism

$$
\operatorname{det} R \Gamma(M, L) \simeq \operatorname{det} R \Gamma\left(M, L\left(-\sum_{i=1}^{r} P_{i}\right) \otimes\left\{\bigotimes_{i=1}^{r} L_{i}\left[P_{i}\right]\right\} .\right.
$$

To see that this is in fact an isometry, one has to show that each term has the Faltings metric on it. This follows from successive application of property 3 . Deleting one point at the time one has the iso- 
morphism:

$$
\begin{aligned}
\operatorname{det} R \Gamma(M, L) & \left.\equiv \operatorname{det} R \Gamma\left(M, L\left(-\sum_{i=1}^{r} P_{i}\right)\right) \underset{i=1}{r} L\left(-P_{i}\right)\left[P_{j}\right]\right]_{i=1}^{r} L\left[P_{i}\right] \\
& \simeq \operatorname{det} R \Gamma\left(M, L\left(-\sum_{i=1}^{r} P_{i}\right)\right){\underset{i=1}{\otimes}}_{\otimes} L\left[P_{i}\right] \otimes_{i=1}^{r} \Theta\left(-P_{i}\right)\left[P_{j}\right] .
\end{aligned}
$$

On $Q\left(-P_{j}\right)\left[P_{j}\right]$ we have the Green's metric, so that the norm on $Q\left(-P_{i}\right)\left[P_{j}\right]$ is given by

$$
\left\|\Theta\left(-P_{i}\right)\left[P_{j}\right]\right\|=\frac{1}{G\left(P_{i}, P_{j}\right)} .
$$

Furthermore, the metric on $\operatorname{det} R \Gamma\left(M, L\left(-\sum_{i=1}^{r} P_{i}\right)\right)$ is isometric with the flat metric on $\mathbb{C}$, because $L\left(-\sum_{i=1}^{r} P_{i}\right)$ is of degree $p-1$. Hence (2.3.29) is indeed an isometry, for the Faltings metric.

The Faltings metric on the space $\operatorname{det} R \Gamma(M, L)$ can now be constructed as follows. Let $U$ be an open covering of $M^{r}$ such that for $\left(P_{1}, \ldots, P_{r}\right) \in U$ the exact sequence (3.2.28) induces the isomorphism

$$
\alpha: \operatorname{det} R \Gamma(M, L) \rightarrow \bigotimes_{i=1}^{r} L\left[P_{i}\right]
$$

(Note that this condition is equivalent with $H^{0}\left(M, L\left(-\sum_{i=1}^{r} P_{i}\right)=H^{1}\left(M, L\left(-\sum_{i=1}^{r} P_{i}\right)\right)=0\right)$. If $\left\{\alpha_{i}\right\}_{i=1}^{r}$ denotes a basis in $H^{0}(M, L)$, then this isomorphism can be given explicitly by

$$
\alpha_{1} \wedge \cdots \wedge \alpha \rightarrow \operatorname{det} \alpha_{i}\left(P_{j}\right) .
$$

From theorem 1 it follows that the map $\psi: M^{r} \rightarrow P i c_{p-1}(M)$, is such that $U \subset \psi^{-1}\left(P i c_{p-1}-\theta\right)$. Moreover the space det $R \Gamma\left(M, L((\mathscr{P}))\right.$ is identified with the fibre over $\psi\left(P_{1}, \ldots, P_{r}\right)$ of $\theta(-\theta)$. Denote by $1_{\theta}$ the unit section of $\theta(-\theta)$ in $\left(P i c_{p-1}-\theta\right.$ and by $\lambda\left(P_{1}, \ldots, P_{r}\right)$ the function on $U$ such that

$$
\lambda\left(P_{1}, \ldots, P_{r}\right)=\left\|\mathbf{1}_{\theta} \psi\left(P_{1}, \ldots, P_{r}\right)\right\|^{2}
$$

Using (2.3.26) and the exact sequence (2.3.28), one obtains the Faltings metric on det $R \Gamma(M, L)$ :

$$
\begin{gathered}
\|\operatorname{det} R \Gamma(M, L)\|_{F}^{2}=\Delta\left\|\mathbb{1}_{\theta} \psi\left(P_{1}, \ldots, P_{r}\right)\right\|^{2} \alpha^{*}\left\|\stackrel{\otimes}{i}=1_{i} L\left[P_{i}\right]\right\|_{F}^{2} \\
=\Delta\left\|\mathbb{1}_{\theta} \psi\left(P_{1}, \ldots, P_{r}\right)\right\|^{2} \frac{1}{\prod_{i \neq j} G\left(P_{i}, P_{j}\right)} \alpha^{*}\left\|_{i=1}^{r} L\left[P_{i}\right]\right\|_{n a t}^{2} \\
=\Delta\left\|\mathbb{1}_{\theta} \psi\left(P_{1}, \ldots, P_{r}\right)\right\|^{2} \frac{1}{\prod_{i \neq j} G\left(P_{i}, P_{j}\right)}\left\|\operatorname{det} \alpha_{i}\left(P_{j}\right)\right\|_{\text {nat }}^{2} .
\end{gathered}
$$

Because this metric is unique up to scalar multiplication, one can use property 4) of theorem 1 to normalize it and describe the proportionality constant $\Delta$ in terms of the invariant $\delta(M)$. Following [20], one first chooses a divisor $D$ on $M$ such that $\Omega(-D)$ is of degree $p-1$. So $D$ is given by: $D=P_{1}+\ldots+P_{p}-Q$. Using the basis $w_{1}, \ldots, w_{p}$ for $H^{0}(M, \Omega)$ introduced earlier, one may derive along similar lines as above the following equality:

$$
\left\|\mathbb{1}_{\theta} \psi\left(P_{1}, \ldots, P_{r}, Q\right)\right\|=\exp (-\delta(M) / 8) \frac{\left\|\operatorname{det} w_{i}\left(P_{j}\right)\right\|_{n a t}}{\prod_{i<j} G\left(P_{j}, P_{k}\right)} \prod_{j} G\left(P_{j}, Q\right) .
$$

(The representation of the proportionality factor in terms of $\delta(M)$ is for convenience; this will be clarified in the next section.) 
In the next section we will generalize the results presented in this section, to compute the metric on the determinant line bundle of the holomorphic $n$-differentials.

\section{ISOMETRIC ISOMORPHISMS OF DETERMINANT LINE BUNDLES ON MODULI SPACE}

In this section we will discuss how the ideas of the previous section generalize to the case of families of Riemann surfaces. Our first concern will be the construction of the determinant line bundle associated to the holomorphic $n$-differentials, and the definition of the Quillen metric on this bundle. This is done in 3.1. Then in (3.2) we introduce the Grothendieck-Riemann-Roch (G-R-R) theorem, and use it to obtain holomorphic isomorphisms between determinant line bundles. As a result we recover the Belavin-Kniznik theorem which states that the string partition function is described by the square of a so-called Mumford form on moduli space.

In (3.3) we will relate the Quillen metric on the determinant bundle of holomorphic $n$-differentials with Arakelov Green's functions using a generalization of the results in 2.3. This leads to an algebraic formulation of the string partition function. We then derive in an algebraic way the modular forms describing the partition function. Finally in (3.4) we discuss the asymptotic behaviour of the partition function.

\subsection{The construction of the determinant line bundle on moduli space}

The determinant line bundle associated to the holomorphic $n$-differentials, can be constructed by varying the conformal structure on the Riemann surface. An infinitesimal variation of the conformal structure is in fact a transformation along a vector in the tangent space of Teichmüller space, hence it corresponds to a traceless deformation of the metric on $M$. Using $(2.2 .2)$ one computes the induced transformation on the differentials $\nabla_{n}^{z}[23]$ :

$$
\delta \nabla_{n}^{z}=\frac{1}{2} \delta g^{z z} \nabla_{z}^{n}+\frac{n}{2} \nabla_{z}^{n}\left(\delta g^{z z}\right)
$$

It is crucial that this variation is holomorphic w.r.t. the complex structure of Teichmüller space. (We recall the fact that locally Teichmüller space is isomorphic to $\mathbb{C}^{3 p-3}$ from which one can prove that $\mathscr{T}_{p}$ is a complex space $[1,10]$.)

The next step is to construct the universal Teichmüller curve $\mathscr{X}_{p}$, which is the natural fibre space over $\mathscr{T}_{p}$ :

$$
\tilde{\pi}: \mathscr{X}_{p} \rightarrow \mathscr{T}_{p} .
$$

It has the property that for $t \in \mathscr{T}_{p}, \tilde{\pi}^{-1}(t)$ (i.e. the restriction to the fibre) is a space $\hat{M}$ isomorphic to the Riemann surface $M$, represented by $t$. The differential

$$
d \tilde{\pi}: T \mathscr{X} \rightarrow T \mathscr{T}_{p} \text { (a tensor of type }(1,0) \text { ) }
$$

has everywhere along the fibre a one-dimensional kernel, which is the tangent of the fibre. So, Ker $d \tilde{\pi}$ defines a line bundle, $\tilde{\Omega}^{-1}$, called the relative tangent line bundle. (Note that this bundle is dual to the bundle of holomorphic quadratic differentials, by virtue of Serre duality). In a similar way one can obtain the relative bundles $\tilde{\Omega}^{\otimes n}$ on $\mathscr{X}_{p}$, to which the operators $\nabla_{n}^{z}$ are associated. By (3.1.1) these bundles vary holomorphic with $t \in \mathscr{T}_{p}$. For each $t \in \mathscr{T}_{p}$ one has

$$
\left(\operatorname{det} R \Gamma\left(M, \Omega^{\otimes n}\right)\right)^{-1}=\left(\wedge^{\max } H^{0}\left(M, \tilde{\Omega}^{\otimes n}\right)\right)^{\star} \otimes\left(\wedge^{\max } H^{1}\left(m, \tilde{\Omega}^{\otimes n}\right)\right)
$$

Using direct images of the projection $\tilde{\pi}$, this yields a holomorphic determinant line bundle over Teichmüller space $\mathscr{\sigma}_{p}$, so one uses $\mathscr{T}_{p}$ as the complex parameter space for the construction of this bundle: $\mathfrak{L}_{n} \rightarrow \mathscr{T}_{p}$. The direct images of the bundles $\tilde{\Omega}^{\otimes n}$ over $\mathcal{X}$ characterize the determinant line bundle $\mathfrak{R}_{n}$ in terms of the cohomology along the fibres of $\mathscr{X}_{p}$ over $\mathscr{T}_{p}$. More precisely, for a sheaf $\mathscr{F}$ defined on $\mathcal{X}$ the higher direct image $R^{i} \tilde{\pi}_{\star} \mathscr{F}$ is the sheaf associated to 


$$
U \rightarrow H^{i}\left(\tilde{\pi}^{-1}(U),\left.\mathscr{F}\right|_{\tilde{\pi}^{-1}}(U)\right), U \subset \mathfrak{N}, i \geqslant 0
$$

on $\pi_{4}\left(R^{0} \tilde{\pi}_{*} \equiv \pi_{*}\right.$ is simply called the direct image).

Using the functorial properties of $R^{i} \tilde{\pi}_{*}$ [29], one can now characterize the determinant line bundle $\mathfrak{l}_{n} \rightarrow \mathscr{T}_{p}$ as

$$
\mathfrak{E}_{n}=\left(\operatorname{det} R \tilde{\pi}_{n} \tilde{\Omega}^{\otimes n}\right)^{-1}=\left(\wedge^{\max } \tilde{\pi}_{\star} \tilde{\Omega}^{\otimes n}\right)^{\star} \otimes\left(\wedge^{\max } R^{1} \tilde{\pi}_{\star} \tilde{\Omega}^{\otimes n}\right)
$$

which by virtue of $(2.3 .9 \mathrm{a}-\mathrm{b})$ reduces to

$$
\begin{aligned}
& \mathfrak{L}_{n}=\left(\wedge^{\max } \tilde{\pi}_{\star} \tilde{\Omega}^{\otimes n}\right)^{\star} n>1 \\
& \mathcal{L}_{1}=\left(\wedge^{P} \tilde{\pi}_{*} \tilde{\Omega}\right)^{*} \otimes R^{1} \tilde{\pi}_{*} \Omega
\end{aligned}
$$

Recall that Teichmüller space is a contractible space so that the line bundle $\mathfrak{L}_{n}$ is trivial. However, as $e_{n}$ carries a nontrivial action of $\Gamma_{p}$, the trivialization cannot be a holomorphic function. Using the fact that the action of $\Gamma_{p}$ extends to $\mathscr{x}$, one may define $\varrho_{n}$ as a holomorphic determinant line bundle over moduli space, by factoring out $\Gamma_{p}$. Hence we have the induced projection

$$
\pi: X \rightarrow \Re
$$

where $X=\mathfrak{X}_{p} / \Gamma_{p}$, the universal curve of moduli space. The corresponding bundle $\mathfrak{C}_{n} \rightarrow \mathfrak{N}$ is thus holomorphically non-trivial.

At this point it is useful to observe that the string partition function is in fact described by a section of the line bundle $G$,

$$
G=\left(\mathscr{L}_{1}\right)^{-d / 2} \otimes \mathscr{L}_{2}
$$

The bundle $\left(\mathscr{L}_{2}\right)^{-1}$ turns out to be the canonical bundle $K$ of moduli space since

$$
\left(\mathcal{L}_{2}\right)^{-1} \equiv \wedge^{3 p-3} \pi_{\star} \Omega^{2}=K_{\Re} \equiv \wedge^{3 p-3} \text { (cotangent space of } \mathfrak{N} \text { ) }
$$

The problem of finding an integrable volume element on $\mathfrak{N}$ has now become a problem of constructing a Hermitean metric on $G$, such that $G$ is holomorphically flat. For this purpose, one can use the Quillen metric. To define the Quillen metric on the holomorphic determinant bundle $e_{n}$, we choose $\left\{\phi_{i}\right\}$ as an basis for $H^{0}\left(M, \tilde{\Omega}^{\otimes n}\right)$. Then a section of $E_{n}$ is given by

$$
s=\left(\phi_{1} \wedge \cdots \wedge \phi_{m}\right)^{-1}, m=(2 n-1)(p-1) \text {. }
$$

The Quillen metric $\|\cdot\|_{Q}$ is defined as [39]

$$
\|s\|_{Q}^{2}=\frac{\operatorname{det} \Delta_{n}}{\operatorname{det}\left(\phi_{i}, \phi_{j}\right)}
$$

where $s$ a section of $\mathfrak{L}_{n}$ and $\Delta_{n}$ is the Laplacian associated with $\nabla_{n}^{z}$ (computed using the admissible metric described in the previous section). Because of (3.1.7) the definition of the Quillen metric on $\mathfrak{f}_{1}$ is

$$
\left\|\left(w_{1} \wedge \cdots \wedge w_{p}\right)^{-1} \otimes \mathbb{1}\right\|_{Q}^{2}=\frac{\operatorname{det}^{\prime} \Delta_{1}}{\operatorname{det}\left(w_{i}, w_{j}\right) \int_{M} \sqrt{g}},
$$

where 1 is the constant section. The Quillen metric is related to the usual $L_{2}$ metric according to [13]

$$
\|\cdot\|_{Q}^{2}=\|\cdot\|_{L_{2}}^{2} \operatorname{det}^{\prime} \Delta_{n}
$$

This metric varies holomorphically on moduli space, and has a unique holomorphic connection whose curvature is given by

$$
\operatorname{Curv\mathfrak {R}_{n}}=\frac{\partial^{2}}{\partial y \bar{\partial} y}, \log \|s\|_{Q}^{2}, y \in \mathfrak{R}, s \in \mathfrak{L}_{n}
$$


which we will write as $\partial \bar{\partial} \log \|s\|_{Q}^{2}$.

Using this metric one can rewrite the partition function

$$
Z_{p}=\int_{\mu}^{3 p} \prod_{i=1}^{-3} d \phi_{i} \wedge d \bar{\phi}_{i} \operatorname{det}\left(w_{i}, w_{j}\right)^{-d / 2} \cdot G
$$

where

$$
G \equiv\left(\frac{\operatorname{det} \Delta_{1}}{\operatorname{det}\left(w_{i}, w_{j}\right) \int_{M} \sqrt{g}}\right)^{-d / 2}\left(\frac{\operatorname{det} \Delta_{2}}{\operatorname{det}\left(\phi_{i}, \phi_{j}\right)}\right)
$$

as

$$
Z_{p}=\int_{\mu}^{3 p} \prod_{i=1}^{-3} d \phi_{i} \wedge d \bar{\phi}_{i} \operatorname{det}\left(w_{i}, w_{j}\right)^{-d / 2} \cdot\left\|s_{2}\right\|_{Q}^{2} \cdot\left\|s_{1}\right\|_{Q}^{-d}
$$

where $s_{1} \in \mathscr{L}_{1}$ and $s_{2} \in \mathfrak{L}_{2}$, are sections given in (3.1.10a), for $m=p$, resp. $m=3 p-3$. (We used the fact that the quadratic differentials $\phi$ on $\Re$ vary holomorphically and may serve as coordinates on $\Re)$. We thus conclude that in order to prove, that the integrand (3.1.13) is really a function on moduli space, one has to prove the existence of a holomorphic isomorphism between $\left(\mathcal{L}_{1}\right)^{-d / 2}$ and $\ell_{2}$ for some value of $d$, which must be an isometry for the Quillen metrics defined on $\mathfrak{L}_{2}$ and $\mathfrak{L}_{1}$.

\subsection{The Grothendieck-Riemann-Roch theorem and the Mumford form on moduli space}

To find the holomorphic isomorphisms among the determinant line bundles over moduli space, requires a generalization of the Riemann Roch theorem to families of Riemann surfaces. This is the Grothendieck-Riemann-Roch theorem. (For a readable description we refer to [29] Appendix A and [31]). It relates the cohomology of the bundles with the intersection theory of the manifold on which the bundles are defined. After we have described shortly the theorem, we apply it to the determinant line bundles $\mathfrak{e}_{n}$, to obtain for $d=26$ the isomorphic isometries describing the integrand in (3.1.13).

To describe the G-R-R theorem, requires the introduction of the Grothendieck groups $K(X)$ (and $K(\mathscr{T})$ ), constructed out of the semi-group $\operatorname{Vect}(X)$ (resp. $\operatorname{Vect}(\Re())$ ) of vector bundles, by dividing out an equivalence relation between these vector bundles. This is called the Grothendieck construction. The Grothendieck relations are those which to each short exact sequence

$$
0 \rightarrow B^{\prime} \rightarrow B \rightarrow B^{\prime \prime} \rightarrow 0, \quad B, B^{\prime}, B^{\prime \prime} \in \operatorname{Vect}(X)
$$

give the relation

$$
[B]=\left[B^{\prime}\right]+\left[B^{\prime \prime}\right] .
$$

The group of isomorphism classes of vector bundles over $X$ modulo these relations, is a free Abelian group, called the Grothendieck group $K(X)$. The relevant thing for us is that the projection $\pi: X \rightarrow \pi$, gives rise to a map $\pi_{!}$defined as

$$
\pi_{!} \equiv \Sigma(-1)^{i} R^{i} \pi_{*}
$$

which is a map for the Grothendieck groups:

$$
\pi_{!}: K(X) \rightarrow K(\mathfrak{T}) \text {. }
$$

The other ingredient in the G-R-R theorem, involves a characterization of vector bundles through their Chern classes. In the context of intersection theory a Chern class $c_{i}$ of a vector bundle over $X$, is a cycle of codimension $i$ on $X$. This is a generalization of an intersection number for two divisors, and is in general a linear combination of varieties of codimension $i$. In this way each Chern class assigns an element of the group of all cycles of codimension $i$, denoted by $A_{i}(X)$, to an element in $K(X)$. The equivalence relation for cycles, induced by the Grothendieck relations for vector bundles, is called 
rational equivalence. For cycles of codimension 1 it is the one induced by the linear equivalence for line bundles, which we discussed earlier.

The Chern character $C h$, is a map

$$
C h: K(X) \rightarrow A(X)
$$

where $A(X)$ is known as the Chow-ring, defined as: $A=\bigoplus_{i} A_{i}(X)$. This is the group of all cycles on $X$ modulo rational equivalence. The Chern character map is a ring homomorphism, and can be expressed in terms of the Chern classes $c_{i}$ [29]:

$$
C h(B)=\operatorname{rank} B+C_{1}(B)+\frac{1}{2}\left(c_{1}^{2}-2 c_{2}\right)(B)+\ldots
$$

( $B$ is here a vector bundle; strictly speaking the argument of $C h$ should be $[B]$, i.e. the element in $K(X)$ determined by $B$ Vect $(X)$ ).

The projection $\pi: X \rightarrow$ induces a map for the Chow ring $A(X)$, also denoted by $\pi_{\star}: A(X) \rightarrow A(\mathfrak{T})$. However, similar to other family index theorems, the maps $\pi_{!}$and $C h$ do not commute. The noncommutativity is precisely the content of the G-R-R theorem. It states that there exists another ring homomorphism $T d$ in $A(X)$ such that the following diagram becomes commutative:

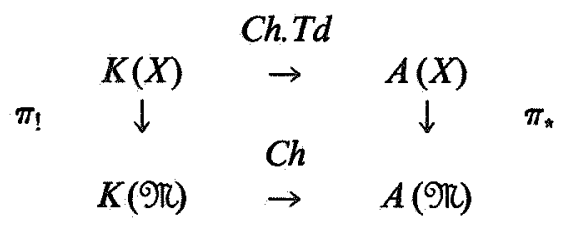

FIGURE 1

More precisely: For the projection $\pi: X \rightarrow \mathfrak{N}$, and a bundle $B \in \operatorname{Vect}(X)$, the Chern character of the projected bundle is given by

$$
\operatorname{Ch}\left(\pi_{!} B\right)=\pi_{\star}\left(C h(B) \cdot \operatorname{Td}\left(\tilde{\Omega}^{-1}\right)\right)
$$

where $\operatorname{Td}\left(\tilde{\Omega}^{-1}\right)$ is the Todd class of the relative tangent bundle $\tilde{\Omega}^{-1}$ of the universal curve $X$. The Todd class can be expressed in terms of Chern classes:

$$
\begin{aligned}
& T d: K(X) \rightarrow A(X) \\
& T d=1+\frac{1}{2} c_{1}+\frac{1}{12}\left(c_{1}^{2}+c_{2}\right) .
\end{aligned}
$$

We now apply (3.2.7) to the case where $B$ is a holomorphic line bundle over $X$. Recalling that $\operatorname{dim} X=\operatorname{dim} \Re+1$, we have upon expanding both sides of (3.2.7):

$$
c_{1}\left(\pi_{!}(B)\right)=\pi_{*}\left[\frac{c_{1}(B)^{2}}{2}-\frac{c_{1}(B) c_{1}(\tilde{\Omega})}{2}+\frac{1}{12} c_{1}(\tilde{\Omega})^{2}\right] .
$$

This formula generalizes the classical Riemann-Roch theorem on a Riemann surface to a family of Riemann surfaces. It characterizes the projected bundle on moduli space by its Chern classes which are elements of the Chow-ring $A(\mathfrak{T})$. In the case of holomorphic line bundles, it thus follows that the projected bundles are determined up to linear equivalence, implying that a possible isomorphism between the bundles over $\pi$ can be chosen holomorphically.

Let us now apply this to the determinant line bundles $\mathfrak{R}_{n}$. One usually puts

$$
c_{1}\left(\mathscr{R}_{1}\right)^{-1} \equiv \lambda=\frac{1}{12} \pi_{*}\left(c_{1}(\tilde{\Omega})\right)^{2}
$$

where we used a property of Chern classes and (3.2.9) for the second equality. The bundle $\left(\mathfrak{L}_{1}\right)^{-1}$ is called the Hodge bundle; $\lambda$ is the Hodge divisor class. Generalizing to $\mathscr{L}_{n}$ one finds Mumford's 
formula (ref. [37] p.101-102).

$$
c_{1}\left(\pi_{\star} \tilde{\Omega}^{\otimes n}\right)=(6 n(n-1)+1) \lambda
$$

which implies that

$$
\mathfrak{e}_{n+1} \simeq\left(\mathfrak{L}_{1}\right)^{6 n^{2}+6 n+1}
$$

are holomorphically isomorphic.

An important property of the above isomorphisms is that on moduli space they are unique up to scalar multiplication. This fact one can use to make contact with the unique curvature forms of the Quillen metric which we defined on the determinant line bundles $\mathfrak{e}_{n}$. It follows that the first Chern class (3.2.11), which is a special element in the Chow-ring $A(\mathfrak{T})$, can be represented by the curvature form of the Quillen metric. This leads to a refinement of (3.2.7) to a formulation of the G-R-R theorem in terms of curvature forms, given in [11]:

$$
c_{1}\left(\mathscr{R}_{n},\|\cdot\|_{Q}\right)=-\int_{X} \operatorname{Ch}(\tilde{\Omega})^{n} T d\left(\tilde{\Omega}^{-1}\right) .
$$

(The minus sign is due to the definition of $e_{n}$ (cf. (3.1.6)).

The 1.h.s. represents the Chern class expressed as the curvature form of the Quillen metric on $\varrho_{n}$, while on the r.h.s. $C h$ and $T d$ are computed using the Hermitean metrics defined on these bundles. Due to this extension of the G-R-R theorem to the level of curvature forms, one concludes that the holomorphic isomorphism is an isometry of the Quillen metrics on the $e_{n}$ 's. In particular, we have for $n=1$ in (3.2.13) the following isometry:

$$
\left(\mathfrak{C}_{1}\right)^{13} \simeq \mathfrak{L}_{2}
$$

Returning to the partition function (3.1.13) one recovers the Belavin-Kniznik theorem [7], since if $d=26$, the integrand takes the form

$$
\frac{\mu(s) \Lambda \mu \overline{(s)}}{\|s\|_{Q}^{2}}, s \in\left(\varrho_{1}\right)^{13}
$$

or, equivalently:

$$
\phi_{1} \wedge \bar{\phi}_{1} \wedge \cdots \wedge \bar{\phi}_{3 p-3}\left|\operatorname{det}\left(w_{i}, w_{j}\right)\right|^{-13}\left\|\left(w_{1} \wedge \cdots \wedge w_{p}\right)^{-13}\left(\phi_{1} \wedge \cdots \wedge \phi_{3 p-3}\right)\right\|_{Q}^{-2}
$$

where $\mu$ in (3.2.15a) is the Mumford isomorphism (3.2.14). So

$$
\left(\left(w_{1} \wedge \cdots \wedge w_{p}\right)^{-13} \cdot\left(\phi_{1} \wedge \cdots \wedge \phi_{3 p-3}\right)^{-1}\right)
$$

is a global holomorphic section of $\mathscr{L}_{2} \otimes \mathscr{L}_{1}^{-13}$ which thus is a modular form w.r.t. $\Gamma_{p}$. This form is referred to as a Mumford form [32].

\subsection{An algebraic formulation of the partition function}

We will now relate the Quillen norm on $\ell_{n}$ with the Faltings metric discussed in section 2. This yields a formulation of (3.2.15b) in terms of Arakelov Green's functions and Faltings' invariant. For this purpose we follow [35] in order to describe a family analogue of theorem 1 .

The first step is to choose a section on the universal curve $X, \sigma: \Re \rightarrow X$, which assigns to each point $y \in \mathscr{R}$ a given point $P$ on a Riemann surface $M$ represented by $\pi^{-1}(y)$. In this way we can make a codimension one, horizontal divisor $D=\sigma(X)$. Using this divisor $D$, we construct a generalization of the function $G(P, Q)$ defined earlier. On each fibre of $\pi$, we have the closed $(1,1)$ form induced by (2.3.11) on the Riemann surface. This constitutes a so-called De Rahm current $[\Phi]$ on $X$. It is a closed $(1,1)$ form on $X$, with distribution coefficients so that $\int_{X}[\Phi]$ is well defined, although $[\Phi]$ itself is not continuous on $X$. One now has the following generalization of the case on a Riemann 
surface (c.f. [18]), namely for every closed form $\alpha$ of degree $\operatorname{dim} X-2$ :

$$
\int_{D} \alpha=\int_{X} \alpha \Lambda[\Phi]=\sigma^{\star} \alpha
$$

where $\sigma^{*}$ is the pull-back of $\sigma$. Subsequently one constructs a function $G\left(P, \sigma^{\circ} \pi(P)\right)$ such that

$$
\partial \bar{\partial} \log G^{2}(P, \sigma \circ \pi(P))=2 \pi i[\Phi]+\text { "smooth terms" }
$$

where the variation on the l.h.s. is along the base, i.e. horizontally. The point $\pi^{\circ} \sigma(P)$ is precisely the point selected on the fibre above $\pi(P)$, so that it does not depend on $P$. The function $G$ has a first order zero along $D$ and is smooth on $X-D$. We can now put a Green's metric on the unit section of the sheaf $O(D)$, by letting

$$
\left\|\mathbf{1}_{Q(D)}\right\|=G\left(P, \pi^{\circ} \sigma(P)\right) \text {. }
$$

Note that the curvature of this metric thus defines a $(1,1)$ form on moduli space. (This is discussed in more detail in appendix A).

Using the exact sequence

$$
0 \rightarrow L(-D) \rightarrow L \rightarrow \sigma^{*} L \rightarrow 0
$$

one deduces before the isomorphism for holomorphic determinant line bundles on moduli space

$$
\operatorname{det} R \pi_{\star} L \simeq \operatorname{det} R \pi_{\star} L(-D) \otimes \sigma^{*} L
$$

To check that this isomorphism is in fact an isometry for the Quillen metrics put on these bundles, we may use the G-R-R theorem in the form (3.2.13). This in fact is a straightforward calculation. We have:

$$
\begin{aligned}
& \operatorname{det} R \pi_{\star} L-\operatorname{det} R \pi_{\star} L(-D) \\
& =\int_{X} \operatorname{Ch}(\Theta(-D))(1-C h(L)) T d\left(\tilde{\Omega}^{-1}\right) .
\end{aligned}
$$

Expanding the r.h.s. yields

$$
\int_{X} c_{1} O(D)\left\{\frac{1}{2} c_{1}(\mathscr{O}(D))-\frac{1}{2} c_{1}(\tilde{\Omega})+c_{1}(L)\right\}
$$

Since $\mathcal{O}(D)$ has the Green's metric, this reduces to, using (3.3.2)

$$
\boldsymbol{\sigma}^{*}\left\{\frac{1}{2} c_{1}(\mathcal{O}(D))-\frac{1}{2} c_{1}(\tilde{\Omega})+\frac{1}{2} c_{1}(L)\right\} .
$$

The first two terms in (3.38) cancel, since $\Omega(-D)$ is of degree $p-1$, which therefore has a flat metric. Hence (3.3.5) is really an isometric isomorphism.

We now take $L$ to be $\tilde{\Omega}^{\otimes n}$. Along similar lines as in the previous section, one constructs the divisor $D$ such that $\tilde{\Omega}^{n}(-D)$ is of degree $p-1$. Appyling (3.3.5) itteratively, one then finds after putting metrics on both sides:

$$
\|s\|_{Q}^{2}=\left\|s^{\prime}\right\|_{Q}^{2} \frac{\prod_{i \neq j} G\left(P_{i}, P_{j}\right)}{\left\|\operatorname{det} \phi_{i}\left(P_{j}\right)\right\|^{2}}
$$

where $s$ is a section of $\mathscr{L}_{n}$, and $s^{\prime}$ a section of the determinant line bundle associated to $\tilde{\Omega}^{\otimes n}(-D)$. This is a direct generalization of (2.3.38).

Because $\tilde{\Omega}^{\otimes n}(-D)$ is of degree $p-1$ by construction, the Quillen metric on the associated determinant bundle is isometric to the usual flat metric on $\mathbb{C}$, and it depends only on the isometry class of $\tilde{\Omega}^{\otimes n}(-D)$. Hence, the Quillen metric on $\operatorname{det} R \pi_{*}\left(\tilde{\Omega}^{\otimes n}(-D)\right.$ can be related to the metric on the unit section of the $\theta$-divisor lifted to the universal curve $[20,35]$. We denote by $\left\|1_{\theta} \psi\left(P_{1}, \ldots, P_{r}\right)\right\|$ the norm of the unit section of $\theta(-\theta)$ lifted to the universal curve. This is a direct generalization of the earlier 
definition (cf. text above (2.3.36)). By virtue of the G-R-R theorem one has the isometric isomorphism:

$$
\operatorname{det} R \pi_{\star}\left(\tilde{\Omega}^{n}(-D)\right) \simeq \pi_{*} \theta(-\theta)
$$

This implies that the Quillen metric $\left\|s^{\prime}\right\|$ in (3.3.9) is proportional to the norm on $\mathbf{1}_{\theta}$, where the proportionality is given by the invariant $\delta$ lifted to moduli space. We will now make this explicit. Choosing an odd theta characteristic, or spin bundle, (which has degree $p-1$ ) on the (spin covering of the) universal curve $X$, one obtains using the G-R-R theorem, the isometry

$$
\left(\mathfrak{e}_{\frac{1}{2}}\right)^{-2} \simeq \mathfrak{L}_{1}
$$

where $\frac{e_{1}}{2}$ is the associated determinant line bundle of $\tilde{\Omega}^{\frac{1}{2}}$. Subsequently, one may prove the following isometric isomorphism

$$
\left(\mathfrak{L}_{\frac{1}{2}}\right)^{8} \simeq\left(\mathfrak{L}_{2}\right)^{-1} \otimes\left(\mathfrak{L}_{1}\right)^{9}
$$

As a result we can express the Quillen norm on $\mathcal{L}_{1}$ entirely in terms of exp $\delta$. First we conclude from (3.3.12) that the norm on $s^{\prime}$ in (3.3.9) is identified with

$$
\left\|s^{\prime}\right\|_{Q}^{2}=\left[\frac{\operatorname{det}^{\prime} \Delta_{1}}{\operatorname{det}\left(w_{i}, w_{j}\right) \int_{\pi^{\prime}(y)} \sqrt{g}}\right]^{-\frac{1}{2}}\left\|1_{\theta} \psi\left(P_{1}, \ldots, P_{p}, Q\right)\right\|^{2} .
$$

This together with the identity (2.3.37) generalized to the universal curve yields, for the Quillen norm on $\mathfrak{L}_{1}$ :

$$
\begin{aligned}
\|s\|_{Q}^{2} & =\left[\frac{\operatorname{det}^{\prime} \Delta_{1}}{\operatorname{det}\left(w_{i}, w_{i}\right) \int_{\pi^{-1}(y)} \sqrt{g}}\right] \\
& =\left[\frac{\operatorname{det}^{\prime} \Delta_{1}}{\operatorname{det}\left(w_{i}, w_{j}\right) \int_{\pi^{-1}(y)} \sqrt{g}}\right]^{-1 / 2} \frac{\prod_{i \neq j} G\left(P_{i}, P_{j}\right)}{\prod_{i} G\left(P_{i}, Q\right)\left\|^{2} \operatorname{det}\left(w_{i}, P_{j}\right)\right\|^{2}}\left\|1_{\theta} \psi\left(P_{1}, \ldots, P_{p}, Q\right)\right\|^{2}
\end{aligned}
$$

So

$$
\frac{\operatorname{det}^{\prime} \Delta_{1}}{\operatorname{det}\left(w_{i}, w_{j}\right) \int_{\pi^{-1}(y)} \sqrt{g}}=\exp \left(\frac{-\delta}{6}\right) .
$$

Furthermore we use (3.3.12) and the above result to obtain the Quillen norm on $\mathscr{L}_{2}$ :

$$
\|s\|_{Q}^{2}=\frac{\operatorname{det}^{\prime} \Delta_{2}}{\operatorname{det}\left(\phi_{i}, \phi_{j}\right)}=\exp \left(\frac{\delta}{12}\right) \frac{\prod_{i \neq j} G\left(P_{i}, P_{j}\right)}{\left\|\operatorname{det} \phi_{i}\left(P_{j}\right)\right\|^{2}}\left\|1_{\theta} \psi\left(P_{1}, \ldots, P_{m}\right)\right\|^{2}
$$

(where $s=\left(\phi_{1} \wedge \cdots \wedge \phi_{3 p-3}\right)^{-1}$ and $\left.m=3 p-3\right)$. It is thus seen that the partition function may be rewritten as

$$
\left|\phi_{1} \wedge \cdots \wedge \phi_{3 p-3}\right|^{2} \exp \left(\frac{9 \delta}{4}\right) \frac{\prod_{i \neq j} G\left(P_{i}, P_{j}\right)}{\left\|\operatorname{det} \phi_{i}\left(P_{j}\right)\right\|^{2}}\left\|1_{\theta} \psi\left(P_{1}, \ldots, P_{m}\right)\right\|^{2} .
$$

This expression is independent of the points $P_{i}$ and of the coordinates $\phi_{i}$ on $\Re$.

The choice of the $\theta$-divisor gives rise to an isomorphism on the universal curve $J a c \simeq P i c_{p-1}$, where $J a c$ denotes the Jacobian. Under this isomorphism the $\theta$-divisor on $P i c_{p-1}$, gives a (symmetric) divisor on the Jacobian. Since the Jacobian is an Abelian variety it is the for a suitable choice of $\theta$, 
the zero-set of the Riemann theta-function. From this one can show that (3.3.15) can be expressed in terms of these functions, so using the parameters of the Siegel upper half plane one can find explicit representations of the Mumford isomorphism describing (3.3.15).

Using the basis $\left(w_{1}, \ldots, w_{p}\right)$ for $H^{0}(M, \Omega)$ one defines a period map $\tau$ which maps Teichmüller space into the Siegel upper half plane $\mathbb{H}_{p}$ :

$\tau: \mathscr{T}_{p} \rightarrow \mathbb{H}_{p}, \tau=\int_{b_{i}} w_{j}$

where

$\int_{a_{i}} w_{j}=\delta_{i j},\left(a_{i}, a_{j}\right)=\left(b_{i}, b_{j}\right)=0,\left(a_{i}, b_{i}\right)=\delta_{i j}$.

The set $\left\{a_{1}, \ldots, a_{p}, b_{1}, \ldots, b_{p}\right\}$ denotes the cycles on $M$; they form a symplectic basis for the first homology group $H_{1}(M, Z)$ specifying the so-called marking of $M$ [27]. The map $\tau$ induces a surjective homomorphism, $\Psi$

$$
\Psi: \Gamma_{p} \rightarrow S p(p, \mathbb{Z})
$$

The group $\operatorname{Sp}(p, \mathbb{Z})$ acts in an analogous way on $\mathbb{H}_{p}$ as $\Gamma_{p}$ does on $\mathfrak{T}_{p}$. The Siegel upper half plane $\mathbb{H}_{p}$ has complex dimension $p(p+1) / 2$ and parametrizes the pairs $\left(A,\left(a_{i}, b_{j}\right)\right)$ where $A$ is a complex algebraic torus corresponding to a point $\mathscr{T} \in \mathbb{H}_{p}$, called an Abelian variety, and $\left(a_{i}, b_{j}\right)$ a basis in $H_{1}(A, \mathbb{Z})$. The space

$$
\mathfrak{Q}=\mathbb{H}_{p} / S p(p, \mathbb{Z})
$$

is called the moduli space of Abelian varieties. In general a pair $\left(A,\left(a_{i}, b_{j}\right)\right.$ does not correspond in a one to one way with a Riemann surface. This is only true up to genus 3 . For higher genus the points in it corresponding to points in $\mathscr{Q}$ form a closed subvariety of dimension 1 for $p=1$ and $3 p-3$ for $p \geqslant 2$.The parameters $\tau$ are local coordinates on $\&$. One may choose $3 p-3$ among them to use as coordinates on $\mathfrak{T}$ however for $p \geqslant 4$ they are no longer independent. In terms of these coordinates the partition function can be written on as $(p>1)$

$$
\left.\left|\phi_{1} \wedge \cdots \wedge \bar{\phi}_{3 p-3}\right| F(\tau)\right|^{-2}(\operatorname{det} \operatorname{Im} \tau)^{-13}
$$

where $F(\tau)$ is a modular form w.r.t. $\Gamma_{p}$, which can be expressed in terms of $\theta$-functions [32,43]. Up to genus 3 one can use relations in the Chow-ring $A^{1}(\mathscr{T})$, obtained by the G-R-R theorem to determine the modular form in (3.3.19) explicity. We will do this in the next sub-section, after we have discussed the asymptotic behaviour of the partition function.

\subsection{The partition function on compacatified moduli space}

We will discuss in this subsection the asymptotic behaviour of the isomorphism (3.2.12). For this purpose it is useful to compactify moduli space. As we will see the asymptotic behaviour of the partition function uniquely determines the modular form in (3.3.19) up to a multiple constant. Subsequently we show that the isomorphism (3.2.12) can be normalized simultaneously for all genera to the value on the genus 1 moduli space.

One can do this by adding the class of Riemann surfaces on which a finite number of simple loops has been contracted to points, called nodes. This is the Deligne-Mumford compactification of $\Re$. The compact space $\overline{\mathscr{T}}$ is called the moduli space of stable curves $[16,37]$. (There are several other compactification mechanisms, for example the Satake compactification; for our purposes however the Deligne-Mumford compactification seems to be the most appropriate one). The boundary $[\Delta]=\bar{\Re}-\mathscr{R}$ is the union of components $[\Delta]=\Delta_{0}, \Delta_{1}, \ldots, \Delta_{p / 2}$, containing surfaces with modes of degree $0 \leqslant i \leqslant[p / 2]$. For $i \neq 0$, the node separates the Riemann surface, for $i=0$ it is non separating. 
An immediate consequence of the compactification is that holomorphic sections of a bundle defined on will generate a pole of a certain order when it is extended to compact moduli space $\bar{\Re}$. The order of the pole is given by its divisor class. It is not possible to extend the bundles $R_{n}$ straightforwardly to $\overline{\mathfrak{T}}$, since $\mathcal{L}_{1}=\left(\operatorname{det} R \pi_{*} \Omega\right)^{-1}$ is no longer a line bundle on $\overline{\mathfrak{R}}$. To remedy this, one considers the regular Abelian differentials $\omega$ instead of $\Omega$, having the property that they generate at most simple poles at the boundary, such that the residues at neighbouring punctures cancel. Away from the nodes they are just holomorphic 1 differentials. More precisely, one denotes the singular set on the universal family $X$ by Sing $[X]$, that is under $\pi: X \rightarrow \Re$, Sing $[X]$ goes over in $[\Delta]$. Choosing a local complex coordinate $t$, we may define the 1-differential $\Omega^{1}$ near the singularity, by

$$
\Omega^{1}=f(t) d t
$$

where $f(t)$ is a regular function, vanishing at $t=0$.

On the other hand following Mumford (ref. [37] p. 101,102) we have denoting $I_{\text {sing }}$ as the ideal of Sing $[X]$

$$
\Omega^{1}=I_{\text {sing }} \cdot \omega \text {. }
$$

That is we have the exact sequence on $X$

$$
0 \rightarrow \Omega^{1} \rightarrow \omega \rightarrow \omega \otimes \theta_{\text {sing }} \rightarrow 0
$$

where $\theta_{\text {sing }}$ is the sheaf of regular functions over $\operatorname{Sing}[X]$ of which $f(t)$ is just a section.

A corollary of the G-R-R theorem, ([37] p.101), is that $\Omega^{1}$ considered over $\bar{X}$, has a non vanishing second Chern class, expressing the fact that $\Omega^{1}$ is no longer a line bundle on the whole of $\bar{X}$. To determine the second Chern class we just use the multiplication property of Chern classes in the above exact sequence:

$$
1+c_{1}(\omega)=\left(1+c_{1}\left(\Omega^{1}\right)+c_{2}(\Omega)\right)(1+0-[\operatorname{Sing}[X])
$$

from which it follows, by equating terms of equal codimension:

$$
\begin{aligned}
& c_{1}(\omega)=c_{1}\left(\Omega^{1}\right) \\
& c_{2}(\Omega)=[\operatorname{Sing}[X]] .
\end{aligned}
$$

The determinant line bundles $\mathfrak{C}_{n}$ are on compact moduli space given by $\mathfrak{R}_{n} \equiv\left(\operatorname{det} R \pi_{\star} \omega^{\otimes n}\right)^{-1}$. Using the modifications (3.4.5) in the G-R-R theorem and putting $c_{1}\left(\left(\mathscr{C}_{1}\right)^{-1}\right)=\lambda$, we find:

$$
\lambda=\frac{1}{12} \pi_{\star}\left(c_{1}(\omega)^{2}+[\operatorname{Sing} X]\right) \text {. }
$$

One calls $\pi_{\star}[\operatorname{Sing}[X]]=\delta$, the compactification divisors class. It is given by [28]

$$
\delta=\Delta_{0}+\frac{1}{2} \Delta_{1}+\ldots+\Delta_{[p / 2]} .
$$

Generalizing to $e_{n}$ one recovers Mumford's formula:

$$
\left.c_{1}\left(\varrho_{n}\right)=-(\lambda+\underset{2}{n})(12 \lambda-\delta)\right)
$$

from which it follows that

$$
e_{n+1} \simeq\left(e_{1}\right)^{6 n^{2}+6 n+1} \otimes O(\Delta)^{-\left(\begin{array}{c}
n+1 \\
2
\end{array}\right)}
$$

is a holomorphic isomorphism. $(\mathscr{O}(\Delta)$ denotes the line bundle on $\overline{\mathfrak{T}}-\mathfrak{T}$, associated to the compactification divisor). As before, the Chern classes can be represented in terms of the curvature forms of the Hermitean metrics on the $\mathfrak{e}_{n}$ over compactified moduli space. This can be used to prove that also on $\mathscr{\mathscr { T }}(3.4 .9)$ is an isometric isomorphism for the Quillen metrics on $\rho_{n}$.

An important property of $\lambda$ and $\delta$ is the factorization property on the components $\Delta_{i \neq 0} \subset \overline{\mathscr{N}}$. This 
is a consequence from the exact sequences on each fibre in $[\operatorname{Sing}[X]]$ over $\Delta_{i \neq 0}$ :

$$
\begin{aligned}
& 0 \rightarrow \omega_{i} \rightarrow \omega \rightarrow \omega_{p-i} \rightarrow 0 \\
& 0 \rightarrow \Omega_{i} \rightarrow \Omega \rightarrow \Omega_{p-1} \rightarrow 0
\end{aligned}
$$

where $i$ denotes the degree of the node. The first sequence implies that $\lambda$ splits into a direct sum on each component, and using the second, the same follows for $\delta$. If then follows that the isomorphisms in (3.4.9) can be normalized to their genus 1 value for all genera simultaneously. This has an interesting consequence for the partition function, when it is extended compact moduli space. One has to be careful with extending the isomorphism describing the partition function to $\bar{\Re}$ since the line bundle $\left(\mathfrak{L}_{2}\right)^{-1}$ is not the canonical bundle of $\overline{\mathfrak{N}}$ :

$$
K_{\overline{\mathscr{N}}}=\Lambda^{3 p-3} T^{*}(\mathfrak{T}) \neq\left(\operatorname{det} R \pi_{\star} \omega^{2}\right)^{-1} .
$$

In fact a short calculation using deformation theory yields (cf. [28] p. 49).

$$
K_{\overline{\mathscr{N}}}=\operatorname{det} R \pi_{\star}(\omega \otimes \Omega)
$$

so

$$
c_{1}(K \bar{\pi})=13 \lambda-2 \delta
$$

while

$$
c_{1}\left(\left(\varrho_{2}\right)^{-1}\right)=13 \lambda-\delta
$$

The global section of $\mathfrak{L}_{1}^{-13} \otimes \mathfrak{L}_{2} \otimes O(-\Delta)$, describing the partition function thus generates a second order pole at the boundary. (We disagree with the results found in $[14,25]$. The partition function has therefore the following asymptotic behaviour:

$$
\phi_{1} \wedge \cdots \wedge \bar{\phi}_{3 p-3}(\operatorname{det} \operatorname{Im} \tau)^{-13}|F(\tau)|^{-2} \sim \frac{1}{|t|^{4}} .
$$

Using the factorization property (3.4.10) we conclude that the string partition function can be normalized for all genera, to its value on $\bar{\pi}_{1,1}$, the moduli space of one punctured tori. In appendix $B$ we will duscuss in general the asymptotic behaviour of the Quillen norms.

In case of genus $p=1,2$, we will derive explicit formulae for the modular form in (3.2.20). For $p=1,(3.4 .6)$ reads $\lambda=\frac{1}{12} \Delta_{0}$ so

$$
\theta(\Delta) \simeq(\pi * \omega)^{\otimes 12}
$$

A section of this bundle is a modular form of weight 12 on $\mathbb{H}_{1}$, which is given by

$$
(\eta(\tau))^{24}=e^{2 \pi i r} \prod_{n=1}^{\infty}\left(1-e^{2 \pi i n \tau}\right)^{24}
$$

It is easily verified since (3.4.17) occurs as the descriminant of an elliptic curve that (3.4.17) is now here vanishing except at $\Delta_{0}$, where it vanishes to order 1 . Together with (3.4.13-14) it follows that

$$
\left(\pi_{!} \omega^{\otimes 2}\right) \simeq\left(\pi_{\star} \omega\right)^{\otimes 14}
$$

Therefore we obtain the well known expression for the integrand (3.3.19):

$$
\frac{|d \tau \wedge \overline{d \tau}|}{\left|\eta(\tau)^{24}\right|^{2}}(\operatorname{Im} \tau)^{-14}
$$

(Note that $\exp \delta(M)=(\eta(\tau))^{24}$ in agreement with [20]). For genus 2, one can derive apart from (3.4.6), a second relation in $A^{1}(\overline{\mathscr{N}})$, (cf. [37]):

$$
10 \lambda=\Delta_{0}+\Delta_{1}
$$


From which it follows that the modular form (3.4.19) is actually a section of

$$
\theta(\Delta) \simeq\left(\Lambda^{2} \pi_{\star} \omega\right)^{\otimes 10}
$$

This section can be expressed analytically as

$$
\mathrm{X}_{10}(\tau)=\left[\prod_{a, b}^{10} \underset{\operatorname{even}}{\boldsymbol{\theta}}\left[\begin{array}{c}
b \\
b
\end{array}\right]\right]^{2}
$$

which vanishes to order 1 at $\Delta_{0}+\Delta_{1}$.

Furthermore from the G-R-R theorem we deduce that

$$
\Lambda^{3}\left(\pi_{\star} \omega^{\otimes 2}\right) \simeq\left(\Lambda^{2} \pi_{*} \omega\right)^{\otimes 3}
$$

so one obtains an expression for (3.3.15)

$$
\frac{\left|d \tau_{11} \wedge d \tau_{12} \wedge d \tau_{22}\right|^{2}}{\left|\chi_{10}\right|^{2}}(\operatorname{det} \operatorname{Im} \tau)^{-13}
$$

Hence we have given an algebraic derivation of some of the results in $[8,36]$. In principal one can derive for arbitrary genus relations like $(3.4 .6,3.4 .20)$ in $A^{1}(\overline{\mathscr{T}})$, to determine explicitly the modular form in (3.3.15). However for genus $p \geqslant 3$ the Hodge divisor class will get contributions also from the interior of $\overline{\mathscr{T}}$, due to the hyperelliptic locus, which makes the calculations more complicated

This concludes our discussion on the analysis of the partition function as a geometric object on the moduli space of stable curves over the complex number field. In appendix $A$ we show that also the conformal anomaly, which we formally still have in $Z$ in (cf. 1.10), has an algebraic geometrical interpretation, such that the holomorphic factorization of the partition function ensures the absence of this anomaly.

\section{THE ARITHMETIC STRUCTURE OF THE STRING PARTITION FUNCTION}

In this section we exhibit a relation between the volume form on moduli space describing the string partition function and algebraic number theory. In particular, it is shown that this volume form can be expressed in terms of the modular or geometric height of the rational points on moduli space defined over an arbitrary algebraic number field.

We begin with explaining in 4.1 what is meant by an arithmetic surface. After presenting some relevant properties of algebraic number fields, we give a more arithmetical description of a Riemann surface, which can be used to describe Riemann surfaces over an arbitrary algebraic number field. Subsequently in 4.2 we introduce the so-called height of a point in a projective space, and we give an outline of Faltings' construction of the modular height, which is a height function on moduli space. Finally in 4.3 we establish a relation between the string partition function and the modular height.

As a result it follows that the "usual" partition function is in fact given by the contribution of the so called infinite primes of $K$ to the modular height. For genus 1 and 2 this will be shown by direct computation. Finally we conjecture a more general string partition function which is a summation involving discrete objects only.

\subsection{Arithmetic surfaces}

A curve $C$ in the projective plane $\mathbb{P}^{2}(\mathbb{C})$ is given by an equation

$$
f(u, v, w)=0 \quad u, v, w \in \mathbb{C}
$$

where $f$ is a homogeneous irreducible form. One can always interpret such a plane curve as a linear projection of a curve living in some higher dimensional projective space $\mathbb{P}^{n}$. This is called a smooth model of $C$. A compact Riemann surface $M(\mathbb{C})$ is just the set of the $\mathbb{C}$-rational points of a plane curve $C$, embedded into this projective space $\mathbb{P}^{n}$. Also the converse is true: any compact Riemann 
surface, can be described as a set of rational points satisfying the polynomial equation defining a curve $C$, embedded into $\mathbb{P}^{n}$. This relation between Riemann surfaces and rational points, remains true for curves defined over any algebraic number field. For example, one can define (4.1) over $\mathbb{Q}$, then the set of rational points corresponds to a surface over $\mathbb{Q}$, called an arithmetic surface.

However, unlike $\mathbf{C}, \mathbf{Q}$ is neither topologically or algebraically closed. To deal with these problems we will need a more abstract definition of an arithmetic surface. This is provided by a description of (arithmetic) surfaces in terms of schemes [29]. A scheme can be thought of as a natural fibre space $M$ with a base formed by the prime ideals of a given ring which we take $\mathbb{Q}$ for the moment. For $\mathbb{Q}$ this base is just the ring of integers of $\mathbb{Q}$, denoted as $\operatorname{Spec}(\mathbb{Z})$. The integral ideals of $\mathbb{Q}$ are the finite fields $\mathbb{F}_{p}$, formed by all integers modulo a prime number $p$. In this way one can specify a curve over $\mathbb{Q}$ by giving the corresponding section of the generic fibre of $\pi: M \rightarrow \operatorname{Spec}(\mathbb{Z})$ over each $\mathbb{F}_{p}$. One calls $\pi: M \rightarrow \operatorname{Spec}(\mathbb{Z})$ the minimal arithmetic surface which corresponds uniquely to the curve $C$ defined over $\mathbb{Q}$. However this surface is not complete, so we need a prescription how to compactify it. We will discuss, by giving some properties of $\mathbb{Q}$, how one constructs a compact arithmetic surface.

The basic ingredient is the fact that on $\mathbb{Q}$ one can define different norms $\mid I_{p}$, indexed by a prime number $p$, called a $p$-adic norm. It is defined as

$$
|x|_{p} \equiv \frac{1}{\left(p^{\text {ord } d_{p} x}\right)}, x \in \mathbb{Q}
$$

where $\operatorname{ord}_{p} x$ is the highest power of $p$ which divides $x$. It can be shown that ||$_{p}$ is a norm on $\mathbb{Q}$, moreover it is a theorem that every non trivial norm on is equivalent to ||$_{p}$ for some prime $p$ or for $p=\infty$ cf. [30]. One denotes the usual norm || inherited from $\mathbb{C}$, by ||$_{\infty}$. The set of all norms ||$_{p}$ on $\mathbb{Q}, p<\infty$, is denoted by $M^{0}$.

The fact that $\mathbb{Q}$ is not algebraically closed, means that not all polynomial equations with coefficients in $\mathbb{Q}$ have solutions in Q. To repair this one must consider an infinite sequence of field extensions of $\mathbb{Q}$ : the union of these is then a closed field. An extension $K$ of $\mathbb{Q}$ is such that every element $\alpha \in K$, satisfies a polynomial equation $x_{0}+x_{1} \alpha+x_{2} \alpha^{2}+\ldots+x_{n} \alpha^{n}=0$, where $x_{i} \in \mathbb{Q}$. The degree of the extension, denoted by $[K: \mathbb{Q}]$, is the degree of the defining polynomial above. For example the set of numbers $a+b \sqrt{2}$ with $a, b \in \mathbb{Q}$ defines an extension of $\mathbb{Q}$ (of degree 2). If $[K: \mathbb{Q}]<\infty$ the extension is algebraic and correspondingly one calls $K$ an algebraic number field. Adding roots of all possible polynomials with coefficients in $\mathbb{Q}$ yields an algebraic closure of $\mathbb{Q}$, denoted by $\overline{\mathbb{Q}}$. Unfortunately the closure of $\mathbb{Q}$ is the union of an infinite number of field extensions. This is very different from $\mathbb{R}$ whose closure is $\mathbb{C}$, which is a finite extension of $\mathbb{R}$ of degree 2 .

The $p$-adic norms defined on $\mathbb{Q}$ can be extended to norms on $K$. One can define a $p$-adic norm \|\|$_{p}$ on $K$ as

$$
\|\boldsymbol{\alpha}\|_{p}=\left|N_{K / \mathbf{Q}}(\alpha)\right| \frac{1^{1}}{[K: \mathbb{Q}]}, \alpha \in K
$$

where $N_{K / Q}$ is called the absolute norm, defined as

$$
N_{K / Q}=(-1)^{n} a_{n}
$$

The $\left\{a_{i}\right\}_{i=0}^{n}$ are the coefficients in the polynomial defining $K$ of which $\alpha$ is a root. We assume that also the other roots $\alpha_{i}$ of this polynomial lie in $K$. It is a theorem (cf. [30]) that (4.1.3) is a norm on $K$ which extends ||$_{p}$ on $\mathbb{Q}$.

Having explored some properties of field extensions of $\mathbb{Q}$, we define an arithmetic surface associated to a curve $C$ over $K$. Denote by $R$ the ring of integers of $K$ and by $\operatorname{Spec}(R)$ its set of all prime ideals. Then there always exists a smooth scheme $M$ together with a morphism $\pi$ such that $\pi: M \rightarrow \operatorname{Spec}(R)$ is a minimal arithmetic surface over $K$, associated to a (non-exceptional) curve $C$ which has its coefficients in $K$. To construct a compact arithmetic surface one may add points at infinity. These points correspond to mappings form $K$ into $\mathbb{C}$. One can do this in $n$ different ways, where $n$ is the degree of the extension: 


$$
\sigma_{i}: K \hookrightarrow \mathbb{C} i=1, \ldots, n ; n=[K: \mathbb{Q}]
$$

The set $\sigma_{i}$ associated to $K$ are called the infinite primes of $K$. Important is, that to each fibre of $\pi$ above an infinite prime $\sigma_{i}$ there corresponds a compact surface associated to the curve $C_{i}=C_{K} \otimes_{\sigma_{i}} \mathrm{C}$. The norm on these fibres above $\sigma_{i}$ is just the usual absolute value $\mid /_{\infty}$. Hence we have $n$ Archimedian norms associated to $n$ infinite primes $\sigma_{i}$, forming a set denoted by $M_{K}^{\infty}$.

\subsection{The height function on moduli space}

In section 3 it was shown that an integrable volume form on moduli space can be constructed using isomorphisms between the determinant line bundles $\mathfrak{e}_{n}$. Such relations define corresponding relations between height functions, which can be used to describe the volume form arithmetically.

It is outside the scope of this paper to give a self contained introduction to theory of height functions, for that we refer to [40] and the references therein. The height $H_{X}(P)$ of a point $P=\left(x_{1}, x_{2}, \ldots, x_{n}\right)$ in the projective space $\mathbb{P}^{n}(K)$ is defined as

$$
H_{K}(P)=\prod_{v \in M_{K}} \max \left\{\left\|x_{0}\right\|_{v},\left\|x_{1}\right\|_{v}, \ldots,\left\|x_{n}\right\|_{v}\right\}
$$

This definition is independent of the coordinate system chosen to parametrize $P$. If $P$ is a point in $P^{n}(\overline{\mathbb{Q}})$ then we define the absolute height $H(P)$ as

$$
H(P)=H_{K}(P)^{1 /[K: Q]}
$$

and the logarithmic height $h(P)$ :

$$
h(P)=\log H(P) \text {. }
$$

These last two definitions have the advantage that they are independent of the field definitions. The height function $h(x), x \in \mathbb{P}^{n}(K)$ satisfies an important property, (see for example [21]), namely that for any constant $c>0$, the number of points $x \in \mathbb{P}^{n}(K)$ with $h(x) \leqslant c$ is finite. To make this intuitively clear, consider the following example [21]. Let $\alpha$ be a point in $\mathbb{P}^{2}(\mathbb{Q})$. Such a point corresponds to a line through the origin in three dimensional space. This line contains at least one (and therefore an infinity) of points with integer coordinates. The logarithmic height $h(\alpha)$, corresponding to $\alpha \in \mathbb{P}^{2}(\mathbb{Q})$ measures the logarithmic distance to the origin of the closest non zero integral lattice point on $\alpha$ :

$$
h(\alpha)=\log \sqrt{\left(a^{2}+b^{2}+c^{2}\right)}
$$

where $(a, b, c)$ is an integral point of the line corresponding to $\alpha$, such that $a, b, c$ have no common factors. It is now clear that for any constant $c>0$ the number of points $\alpha \in \mathbb{P}^{2}(\mathbb{Q})$ with $h(\alpha) \leqslant c$ is finite. One says that the height function $h(\alpha)$ measures the "arithmetic complexity" of the point $\alpha$.

The definition of the height can be generalized to an arithmetic surface $\pi: M \rightarrow \operatorname{Spec}(R)$ associated to a curve $C$ over $K$. Spec $(R)$ is projective, so it can be embedded it into some projective space $\mathbb{P}^{n}(K)$. Once we have fixed such an embedding, any point on the curve (or on the arithmetic surface) corresponds uniquely to a point in $\mathbb{P}^{n}(K)$. That is, there exists for any point $x \in \mathbb{P}^{n}(K)$ a map

$$
\phi: \operatorname{Spec}(R) \rightarrow \mathbb{P}_{R}^{n}(K) \text {. }
$$

On $P_{R}^{n}(K)$ we have the constant bundle $O(1)$ generated by the global sections $x_{1}, \ldots, x_{n}$ which are projective coordinates on $\mathbb{P}^{n}$. The pull-back $\phi^{*} O(1)$ becomes a line bundle over $\operatorname{Spec}(R)$, One can now prove that for a point $x \in \mathbb{P}^{n}(K)$, the height $h(x)$ can be given by

$$
h(x)=\frac{1}{[K: \mathbb{Q}]} \operatorname{deg} \phi^{\star} \mathscr{Q}(1)
$$

where deg denotes the degree of the line bundle. In fact we may generalize (4.2.6) to the case of a (very ample) line bundle $\mathcal{L}$ on $M$ such that (4.2.5) corresponds to $\mathcal{L}$ (i.e. the sections of $\mathcal{L}$ serve as 
projective coordinates). Then $\phi^{*}(\mathcal{L})$ is a line bundle over $\operatorname{Spec}(R)$. Correspondingly one has for each point $x \in M(K)$ the height function associated to $\mathcal{C}$ :

$$
h_{\mathfrak{L}}(x)=\frac{1}{[K: \mathbb{Q}]} \operatorname{deg}\left(\phi^{*} \mathfrak{L}\right) \text {. }
$$

The degree of a line bundle over $\operatorname{Spec}(R)$ can be computed using a metric on this bundle. Putting a metric on a line bundle over $\operatorname{Spec}(R)$, turns it into what is called a metrized line bundle over $\operatorname{Spec}(R)$ which can be described as follows. Suppose we have a regular curve $C$ over $\operatorname{Spec}(R)$, and suppose we have for each infinite prime $\sigma_{i}$ a Hermitean metric on the Riemann surface associated to $\sigma_{i}$. A metrized line bundle $\mathcal{E}$ on the arithmetic surface $M$ is a line bundle together with a Hermitean metric \|\|$_{\infty}$ on the line bundle $\mathcal{L}_{i}$ induced by $\mathcal{L}$ on each Riemann surface associated with the infinite prime $\sigma_{i}$. Such a line bundle will be denoted by $\mathcal{L} \otimes_{\sigma_{i}} C$. This definition is essentially coordinate free, therefore we may choose a metric which is suitable for our purposes. We will choose the metrics on each $\varrho_{i}$ such that they are admissible w.r.t. the metric on the Riemann surface associated to $\sigma_{i}$. One calls the metrized line bundle admissible if each $\mathfrak{C}_{i}$ is admissible.

Such an admissible line bundle $\mathcal{E}$ is isometric to a line bundle $\mathcal{O}(D)$, where $D$ is the so called Arakelov divisor of a section of $L$ on the arithmetic surface. We refer the reader to [15] pp $293 \mathrm{ff}$. and to [21] for an introduction to Arakelov's intersection theory. Here we will simply quote the result explained there, namely that under the pull back of the section $\sigma: \operatorname{Spec}(R) \rightarrow M$ of $\pi: M \rightarrow \operatorname{Spec}(R)$ the line bundle $L=\sigma^{*} \mathcal{L}$ becomes a metrized line bundle over $\operatorname{Spec}(R)$, (i.e. a projective rank one $R$ module) of which the degree is given by

$$
\operatorname{deg}(L)=\log (\operatorname{order} L / R \cdot s)-\sum_{M_{\kappa}^{\infty}} \log \|s\|_{i},
$$

where $s$ is a section of $L$ and $\|\cdot\|_{i}$ denotes the Hermitean metric corresponding with the infinite prime $\sigma_{i}$ (Note that this definition is consistent with 2.1.4). If $L$ is free, then we can take a generating section $s \in L$ such that $L=R \cdot s$ so

$$
\operatorname{deg}(L)=-\sum_{M_{k}^{\infty}} \log \|s\|_{i}
$$

and in particular when $R=\mathbb{Z}$ we have (up to a sign)

$$
\operatorname{deg}(L)=-\log |s|_{\infty} \text {. }
$$

Using (4.2.7-8), one can define a height function on moduli space. Consider a fibration of (principally polarized) Abelian varieties defined over $\mathbb{R}$ of genus $p$ :

$$
p: A \rightarrow \mathbb{Q}(\overline{\mathbb{Q}})
$$

where $\mathscr{Q}$ is the moduli space of Abelian varieties of given genus over $\overline{\mathbb{Q}}$. Denote by $\Omega_{A / \mathscr{Q}}$ the cotangent bundle of the fibration. Faltings used the bundle $L \equiv\left(\operatorname{det} R p_{\star} \Omega_{A / Q}\right)^{n}$ on $Q(\bar{Q})$ to define an embedding of $\mathbb{Q}$ into some projective space $\mathbb{P}^{r}(\overline{\mathbb{Q}})$ of dimension $r$. Next we put for each infinite prime $\sigma_{i}$ an admissible metric on the bundle $\Omega \otimes_{\sigma_{1}} \mathbf{C}$. By the results explained in the previous sections we therefore have a unique Hermitean metric (up to scalar multiplication) on $L \otimes_{\sigma_{1}} \mathrm{C}$, of which the curvature is given by $\partial \bar{\partial} \log \|s\|^{2}$. Then one may define a height function $h_{L}$ on $\mathscr{Q}(\overline{\mathbb{Q}})$, such that for a number field $K$ there are only finitely many points $x \in \mathcal{Q}(K)$ with $h_{L}(x) \leqslant c$, for any constant $c$. A direct computation of $h_{L}(x)$ is very complicated but it is possible to compute it in a way that does not depend on the bundle $L$ over $\mathcal{Q}$. For this purpose one considers the modular height $h(A)$.

Let $x$ be the $K$-rational point in $\mathscr{Q}(K)$, defined by a (semi-stable principally polarized) Abelian variety $A$ over $K$, and consider (the connected component of) its Neron model $A^{o}$ over $R$ :

$$
\pi: A^{o} \rightarrow \operatorname{Spec}(R)
$$

The bundle $\Omega_{A / R}$ has a Hermitean metric for each infinite prime. Let $\omega_{A / R} \equiv \operatorname{det} R \pi_{\star} \Omega_{A / R}$ which thus 
is a metrized line bundle over $\operatorname{Spec}(R)$, (i.e. a projective rank one $R$-module). One now defines the modular height $(A)$ :

$$
h(A)=\frac{1}{[K: \mathbb{Q}]} \operatorname{deg}\left(\omega_{A / R}\right)
$$

which can be computed using (4.2.8). This definition is independent of the field extension of $K$. The following theorem relates $h(A)$ with $h_{L}(x)$ :

THEOREM 2 (Faltings [21]). There exists a constant $c$ independent of the number field $K$ and independent of $A$, such that

$$
\left|h_{L}(x)-n h(A)\right| \leqslant c, \quad x \in \mathbb{Q}(K)
$$

where $n$ is the power of det $R \pi_{*} \Omega_{A / \Theta}$ defining $L$.

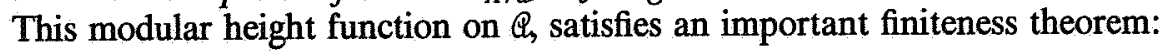

THEOREM 3 (Faltings [21]). Let $K$ be a number field and fix a constant $c>0$. Then up to isomorphism, there exist only finitely many $K$-rational points on $\mathbb{Q}(K)$ defined by principally polarized Abelian varieties of given genus over $K$, such that $h(A) \leqslant c$.

\subsection{The relation between the modular height and the string partition function}

With the results in (4.2) we can now explain the relation between $h(A)$ and the volume form on moduli space describing the string partition function. We will compute $h(A)$, for Abelian varieties over $K$ of genus 1 resp. 2, using the theory of modular forms. Recall that in case of genus 1 resp. 2, the $K$ rational points are in direct correspondence to curves over $K$ of genus 1 resp. 2 . In fact the computation of $h(A)$ in the genus 1 case is standard and can be found in e.g. [40]. In appendix $\mathrm{C}$ we have given the computations for both cases. One finds for a genus 1 stable curve $C / K$ over $K$ :

$h(C / K)=\frac{1}{12[K: \mathbb{Q}]}\left[\log \left(\left|N_{K / \mathbb{Q}} \Delta_{C / K}\right|\right)-\sum_{M_{K}^{\infty}} \epsilon_{i} \log \left(\left|\Delta\left(\tau_{i}\right)\right|\left(I m \tau_{i}\right)^{6}\right)\right]$

where $N_{K / 0}$ is defined in (4.1.4), $\Delta_{C / K}$ the minimal discriminant of $C / K, \tau_{i}$ the modular parameter associated with the infinite prime $\sigma_{i}$ and $\Delta\left(\tau_{i}\right)$ the cusp form of weight 12. (The parameter $\epsilon_{i}=1,2$ depending on $\sigma_{i}$ see [40]). For a genus 2 stable curve $C / K$ one finds

$h(C / K)=\frac{1}{10[K: \mathbb{Q}]}\left[\log \left(\left|N_{K / \mathbb{Q}} \Delta_{C / R}\right|\right)-\sum_{M_{K}^{\Phi}} \epsilon_{i} \log \left(\left|\chi_{10}\left(\tau_{i}\right)\right|\left(\operatorname{det} \operatorname{Im} \tau_{i}\right)^{5}\right)\right]$

where $\chi_{10}\left(\tau_{i}\right)$ is the cusp form of weight 10 , and $\tau_{i}$ the period matrix. From these results one thus concludes that the integral of the string partition function for genus 1 resp. genus 2 is given by the contribution of each infinite prime of $K$ to the modular height, (cf. (3.3.21)) that is, at each infinite prime, we have that

$$
e^{-2 h_{\infty}(C / K)} d \tau \Lambda \overline{d \tau} \quad \text { for genus } 1
$$

and

$$
e^{-2 h_{\omega}(C / K)} d \tau_{11} \wedge \cdots \wedge \overline{d \tau_{22}} \text { for genus } 2
$$

are volume forms on the moduli space of genus 1 resp. 2 stable curves for each infinite prime $\sigma_{i}$. $h_{\infty}(C / K)$ denotes the contribution of the infinite primes to $h(C / K)$. Fixing an infinite prime, one thus recovers the partition function, for genus 1,2 .

For higher genus, $p \geqslant 3$, it is not possible to compute the height as is done is appendix C. However, the conclusion in (4.3.3) can be generalized to arbitrary genus as follows. Recall, from section 3 that the partition function has for arbitrary genus $\geqslant 2$ the form (cf. (3.2.15a-b)) 


$$
\frac{\mu(s) \wedge \overline{\mu(s)}}{\|s\|_{Q}^{2}}
$$

where $s$ is a section of $\left(\varrho_{1}\right)^{13}$, and $\mu$ is the Mumford isomorphism:

$$
(\operatorname{det} R \Omega \omega)^{13} \underset{\mu}{\sim} \operatorname{det} R \pi_{*}\left(\omega^{2}\right) \otimes \mathcal{O}(-2 \Delta) .
$$

To avoid unnecessary technical details we consider a $\mathbb{Q}$ rational point in the moduli space. Furthermore we consider the bundle $\mathcal{L}_{1}$ over $\mathbb{Z}$, which makes it a (projective) rank $1 \mathbb{Z}$-module of which $s$ is a section. It can be shown that the Mumford isomorphism (4.3.5) is also given over $\mathbb{Z}$ [19]. Now we take vectors $t_{1}, \ldots, t_{3 p-3}$ together with their complex conjugates such that $\tilde{s}=\left(t_{1} \wedge \cdots \wedge t_{3 p-3}\right)^{-1}$ is a section of the line bundle $\Lambda^{\max } T(\overline{\mathscr{T}})$ taken over $\mathbb{Z}$, (so $\tilde{s}$ has a double zero at the boundary of $\overline{\mathfrak{T}}$ ). Acting with (4.3.4) yields the height of the $\mathbb{Q}$-rational point $x$ corresponding to a curve $C$ :

$$
\frac{|\mu(s) \cdot \tilde{s}|^{2}}{\|s\|^{2}}=e^{26 h(x)}
$$

(The factor 26 is, because we took a section of $e_{1}$ instead of $\left(\varrho_{1}\right)^{13}$ ). If $s$ is a generating section, the contribution from the finite primes to the height vanishes (cf. (4.2.10)) so the r.h.s. of (4.3.6) reduces to a product over the contributions of all infinite primes. (Fixing an infinite prime leads for the case of genus 1,2 to the partition function (4.3.3)).

In respect of (4.3.6) it seems natural to define the string partition function as

$$
Z=\lim _{K \in \mathbb{Q}(K)} \sum_{x)} \exp (26 h(x))
$$

where the limit is over all algebraic number fields $K$ and the summation is over the $K$-rational points in $\mathcal{Q}$. In this way the integral over moduli space of the usual partition function, is a special case of the formula above. Note that for bounded height this is a finite summation. In (4.3.7) one should incorporate a weight for each rational point. This requires knowledge on the density of the $K$-rational points in moduli space, which is not available at the moment. On the other hand it is known that for genus larger than 23, moduli space is of general type [28]. The higher dimensional Mordell conjecture (cf. [45]) would then imply that the number of rational points in (4.3.7) is essentially finite, so (4.3.7) would have stranger convergence properties than the usual integral over $\mathfrak{T}$.

Another aspect of (4.3.7) is that it might be used as a regularization by fixing a large enough number field and an upper bound for the height. For example in the case of genus 1, one can use Tate's algorithm [41] to calculate the discriminant $\Delta_{C / K}$ in (4.18) and numerical integration to compute the periods $\tau_{i}$. Thus one can determine the height numerically to any desired accuracy. In a subsequent paper [19] we will discuss in more detail the computation of (4.3.6), in the case when other lattices are used.

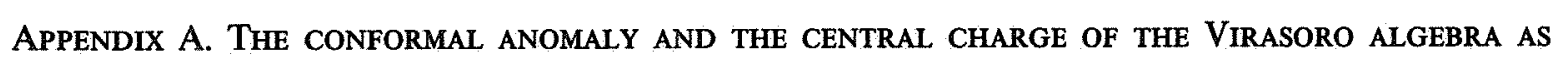
GEOMETRICAL OBJECTS ON MODULI SPACE

In this appendix we relate the conformal anomaly and the central charge of the Virasoro algebra with the Weil Petersson geometry of moduli space.

Denote by $\hat{g}$ the constant curvature metric on a Riemann surface $M$, obtained by a conformal transformation of the Arakelov metric $\hat{g}=e^{\sigma} g$, where $\sigma$ is the conformal factor. Furthermore let $\|s\|_{Q, \sigma}$ be in the Quillen norm of a section $s$ of the bundle $\mathfrak{L}_{n}$ over $\Re$, computed with $\hat{g}$. Then the $\sigma$ dependence of the Quillen metric is given by $[2,3]$

$$
\frac{\|s\|_{Q, \sigma}}{\|s\|_{Q, A}}=\exp -\left[\frac{(6 n(n-1)+1)}{24 \pi^{2}}\right] \int_{\pi^{-}(y)}\left(R e^{\sigma}+\partial \sigma \wedge \bar{\partial} \sigma\right), y \in \mathscr{N}
$$

where $\|s\|_{Q, A}$ is the Quillen metric computed with the Arakelov metric $g$ and $R$ the curvature $(1,1)$ 
form of $g$. Expression (A.1) is called the conformal anomaly. The integral on the r.h.s. is referred to as the Liouville action associated with $g$. Observe that the Quillen metric of the bundle $G=\left(\mathscr{L}_{1}\right)^{-13} \otimes \mathscr{L}_{2}$ is independent of $\sigma$, so that the string partition function is conformally invariant, in $d=26$ dimensions.

The Weil Petersson metric is defined by the Hermitean product [47]:

$$
<\psi, \phi>=\int_{M} \frac{\psi \bar{\phi}}{\lambda^{2}}
$$

where $\phi, \psi$ are holomorphic quadratic differentials, and $\lambda$ is the line element of the constant curvature metric $\hat{g}$ on the Riemann surface $M$. This metric defines a complete, Kähler metric, the so called Weil-Petersson metric, on compactified moduli space $\bar{\Re}$ [47]. Its Kähler $(1,1)$ form $\bar{\omega}_{w p}$ is an element of the Chow-ring $A(\mathfrak{T})$ represented by the curvature of the metric (induced by $\hat{g}$ on $M$ ) on a positive line bundle. In fact one has the equality on the level of forms [48]

$$
\frac{1}{2 \pi^{2}} \bar{\omega}_{w p}=\pi_{*} c_{1}\left(\omega^{2}\right)
$$

Using this, one has the following expression for the first Chern class of the Quillen metric $\|\cdot\|_{Q, \sigma}$ on $e_{n}$ :

$c_{n}\left(\varrho_{n},\|\cdot\|_{Q, \sigma}\right)=\left[\frac{6 n(n-1)+1}{24 \pi^{2}}\right] \bar{\omega}_{w p}+\frac{1}{12} \delta$

where $\delta=\pi_{\star} c_{2}(\Omega)$ (cf. (3.4.7)). taking $n=1$, and combining with (3.3.15a) we find the relation

$$
\frac{1}{2 \pi^{2}} \bar{\omega}_{w p}+\delta=\partial \bar{\partial}\left[-\frac{1}{\pi^{2}} \int \operatorname{Re}^{\sigma}+\partial \sigma \wedge \bar{\partial} \sigma+\frac{1}{i \pi} \delta(M)\right]
$$

where $\delta(M)$ is Faltings' invariant. For genus 1 this reduces to (cf. (3.4.16-19)):

$$
\frac{1}{2 \pi^{2}} \bar{\omega}_{w p}=\pi_{\star} c_{2}(\Omega)=\frac{d \tau \wedge \overline{d \tau}}{|\eta(\tau)|^{24}}
$$

This result has an interesting property for the central charge in the Virasoro algebra. which is generated by the energy momentum tensor $T$, defined as

$$
T \equiv \delta_{g} S[x, g]
$$

where $S[x, g]$ is the action of the theory, varied w.r.t. the metric on the Riemann surface. The conformal variations of $g$, give rise to an expression of the conformal anomaly in terms of the two point correlation function $<T(z) T(w)>$ (cf. [9]):

$$
\lim _{z \rightarrow w}\left\{T(z) T(w)>=\frac{c}{24}\right.
$$

where $c$ is the central charge in the Virasoro algebra. On the other hand by virtue of the Teichmüller variations in (A7), one can consider the energy momentum tensor as a connection in some holomorphic line bundle $\mathfrak{L}_{T}$ over moduli space, carrying a Hermitean metric $\|\cdot\|_{c}$ induced by $\hat{g}$ on $M$ (cf. [24])

$$
<T>=\partial \log \|s\|_{c}^{2} .
$$

Then the two point correlation $\langle T \bar{T}>$ yields an expression for the obstruction to the holomorphic factorization on moduli space

$<T \bar{T}>_{c o n} \equiv<T \bar{T}>-<T><\bar{T}>=\partial \bar{\partial} \log \|s\|_{c}^{2}$.

By the above results this is just another expression for the conformal anomaly. In order to relate (A8) with (A10) it suffices to, by virtue of the factorization property (3.4.10) on degenerate surfaces, to 
integrate (A6) over $\overline{\mathscr{T}}_{1,1}$, the moduli space of once punctured tori. One finds

$$
\int_{\pi_{4,1}} c_{1}\left(\mathscr{L}_{1}\|\cdot\|_{Q, \sigma}\right)=\int_{\pi_{4,1}} \frac{d \tau \wedge d \bar{\tau}}{|\eta(T)|^{24}}=\frac{1}{12}
$$

where we have used that $(\eta(\tau))^{24}$ is a modular form of weight 12 . (This is an alternative derivation of the result found in [42]).

Consequently, we have, if $\mathcal{L}_{1}$ carries the Quillen metric $\|\cdot\|_{Q, \sigma}$, the isometry

$$
\mathfrak{e}_{T} \simeq\left(\mathscr{L}_{1}\right)^{c / 2}
$$

This is in fact a generalization of the isomorphism (3.2.14), showing that the matter content of a two dimensional conformal field theory can be expressed as a power of the Hodge bundle. This power is given by the central charge of the Virasovo algebra of the energy momentum tensor. In this respect, one can view the bosonic string theory embedded in flat Euclidean space as a special case of (A.12) where $c=26$ and $\mathscr{L}_{T} \simeq K_{\Re}$.

Furthermore, because of (A.11), it follows that $\varrho_{T}$ is a true (holomorphic) line bundle, i.e. admitting (holomorphic) sections if and only if the central charge satisfies $\frac{c}{24} \in \mathbb{Z}$. (We disagree at this point with the result found in [24]). This condition is not met in bosonic string theory, which implies that in this theory $\mathfrak{L}_{T}$ is a projective bundle [27].

\section{APPENDIX B: ASYMPTOTIC EXPANSIONS OF LAPLACIANS}

In this appendix we will give asymptotic expansions for the determinants $\operatorname{det} \Delta_{n}$, using the constant curvature metric (cf. appendix A). We will do this for nodes of degree $i>0$ on a Riemann surface. The relation between the Quillen norm and the Faltings norm using the constant curvature metric on $M$ is slightly modified w.r.t. the result presented in section 3:

$$
\begin{aligned}
\|s\|_{Q, \sigma}^{2}=\frac{\operatorname{det} \Delta_{n}}{\operatorname{det}\left(\phi_{i}, \phi_{j}\right)} & = \\
& =\frac{\left(\frac{\operatorname{det} \Delta_{1}}{\operatorname{det}\left(w_{i}, w_{j}\right)}\right)^{-\frac{1}{2}}\left\|1_{\theta}, \psi\left(P_{1}, \ldots, P_{m}\right)\right\|^{2} \prod_{i \neq j} G\left(P_{i}, P_{j}\right)}{\left\|\operatorname{det} \phi_{i}\left(P_{j}\right)\right\|_{\text {nat }}^{2} \prod_{i} G_{R}\left(P_{i}\right)}
\end{aligned}
$$

where $G(P, Q)$ is the exponential of the Green's function of the constant curvature metric satisfying

$$
\begin{aligned}
& \partial \bar{\partial} \log G^{2}(P, Q)=-\pi \delta(P, Q)+\frac{\pi}{A} \hat{g}(P) \\
& \int_{M} \sqrt{\hat{g}} \log G(P, Q)=0
\end{aligned}
$$

and where $A$ is the area: $A=\int_{M} \hat{g}$. The function $G_{R}(P)$ in (B.1) is defined as

$$
G_{R}(P)=\lim _{Q \rightarrow P} \frac{G(P, Q)}{|z(P)-z(Q)|^{2} \hat{g}(P)} .
$$

Note that this function is just unity in the Arakelov metric. (We will not go into the derivation of (B.1); the factor $G_{R}(P)$ arises naturally in chiral bosonization cf. [43]). The advantage of the constant curvature metric is that one can use the formulae of [33] to find the asymptotic behaviour of (B.1). For this purpose we consider a surface with a node of degree $i>0$, and divide it in into three parts, two parts away from the node having area $8 \pi(i-1)$ and $8 \pi(p-i+1)$ respectively and an anulus $A_{t}$ around the node with area $8 \pi$. Furthermore we take the points $P_{i}$ in (B.1) such that we have $2 n-1$ 
one of them on $A_{t}$. The asymptotic analysis of the determinants can then be restricted to the anulus. The Poincaré metric on the anulus is given by

$$
g_{t}^{\star}=\frac{2 a^{2}}{|w|^{2}} \frac{1}{\cos ^{2}(a \log |w|)}
$$

where $t$ is the transversal coordinate, measuring the radius of the anulus and $w$ is a coordinate. The parameter $a$ is given by the condition that $\int_{A_{t}} \sqrt{g_{t}^{*}}=8 \pi$. In [33] it was shown that there exists a constant $C$ such that on $A_{t}$

$$
\frac{1}{C} g_{t}^{*}<\hat{g}_{t}<C g_{t}^{*} \forall t
$$

The Green's function $g(P, Q)=\log G(P, Q)$ on $A_{t}$ is given by

$$
\begin{gathered}
g(P, Q)=\log |z(P)-z(Q)|^{2}-\frac{1}{8 \pi} \int d^{2} w \sqrt{\left(\hat{g}_{t}(w)\right)}\left(\log |z(P)-w|^{2}+\log \mid z(Q)-w^{2}\right)( \\
+\frac{1}{64 \pi^{2}} \int d^{2} w d^{2} w^{\prime} \sqrt{\left(\hat{g}_{t}(w)\right)} \sqrt{\left(\hat{g}_{t}\left(w^{\prime}\right)\right)} \log \left|w-w^{\prime}\right|^{2}
\end{gathered}
$$

Furthermore the holomorphic $n$-differentials $\phi_{1}$ behave near separating nodes according to (c.f. [33])

$$
\phi_{i} \sim \frac{1}{t^{i}}(d t)^{j} i=1, \ldots, 2 n-1 .
$$

It is now straightforward to find the following asymptotic expansions of determinants

$$
\begin{aligned}
\operatorname{det} \Delta_{n} & \sim|t|^{1 / 6}(\log |t|)^{2 n-1} \quad n=2,3, \ldots \\
\operatorname{det} \Delta_{1} & \sim|t|^{1 / 6} \\
\operatorname{det} \Delta_{n} & \sim|t|^{-1 / 12} . \quad n=\frac{1}{2}, \frac{3}{2}, \ldots
\end{aligned}
$$

(These results agree with computations using the Zelberg zeta function see e.g. [17]).

The Weil Petersson volume element on moduli space can be extended smoothly to the boundary. Using the parametrization of the quadratic differentials as in [33] one has

$$
\frac{\phi_{1} \wedge \cdots \bar{\phi}_{3 p-3}}{\left|\operatorname{det}\left(\phi_{i}, \phi_{j}\right)\right|} \sim \frac{1}{|t|^{2}(\log |1 / t|)^{3}} .
$$

Subsequently we make use of the results of [33] to obtain

$$
\operatorname{det}\left(w_{i}, w_{j}\right) \sim \text { const }
$$

at nodes with degree $i>0$. Putting all together one finds for the asymptotic behaviour of the string partition function

$$
\begin{aligned}
& \phi_{1} \wedge \cdots \wedge \bar{\phi}_{3 p-3}\left(\frac{\operatorname{det}^{\prime} \Delta_{1}}{\left|\operatorname{det}\left(w_{i}, w_{j}\right)\right|}\right)^{-13}\left(\frac{\operatorname{det}^{\prime} \Delta_{2}}{\left|\operatorname{det}\left(\phi_{i} \phi_{j}\right)\right|}\right) \\
& \left|\left(\operatorname{det} w_{i} w_{j}\right)\right|^{-13} \sim \frac{1}{|t|^{4}}
\end{aligned}
$$


APPENDIX C: THE HEIGHT OF GENUS 1 AND 2 CURVES $^{*}$ )

Let $K$ be a number field (i.e. $[K: Q]<\infty), R$ its ring of integers, $S:=\operatorname{Spec}(R)$. Let $C$ be a curve over $K$ having stable reduction, let $C \stackrel{\pi}{\rightarrow} S$ be its stable model. The height of $C$ is defined as: $h(C)=\frac{1}{[K: Q]} \operatorname{deg}\left(\operatorname{det} \pi_{\star} \omega_{C / S}\right)$, where $\omega_{C / S}$ is the invertible $\theta_{C}$-module of regular relative differentials. Recall that if $\mathcal{E}$ is a metrized invertible $\theta_{S}$-module we have:

$$
\operatorname{deg} \mathcal{L}=-\sum_{\nu \in S} \epsilon_{v} \cdot \log \|s\|_{\nu}=-\sum_{\nu \in S_{\text {fin }}} \log \|s\|_{\nu}-\sum_{\nu \in S_{\infty}} \epsilon_{\nu} \cdot \log \|s\|_{v},
$$

where $s$ is an arbitrary nonzero meromorphic section of $e$ So in order to compute $h(C)$ we need a nonzero global differential form on $C$. We do not want the local contributions to depend on an arbitrary choice of such a form. This can be achieved by using modular forms.

The genus 1 case

Suppose that $C$ is of genus 1 , with a given point called " 0 " (i.e. $C$ is elliptic). A canonical nonzero element $\Delta$ of $H^{0}\left(C, \omega_{C / K}\right)^{\otimes 12}$. is constructed as follows: Let $0 \neq \omega H^{0}\left(C, \omega_{C / K}\right)$, let $t$ be a local coordinate at 0 such that $\omega=(1+\ldots)$. dt, let $x$ be the unique regular function on $C-\{0\}$ such that at $0: x=\frac{1}{2}+\ldots$, let $R$ be the set of points on $C$ where $d x$ has a zero, $(\# R=3)$. Then define

$$
\Delta(C, \omega):=\prod_{P \neq 0}(x(P)-x(Q)),
$$

and $\Delta:=\Delta(C, \omega) \omega^{12}$. The reader can check that $\Delta(C, \lambda \omega)=\Delta(C, \omega) \lambda^{-12}$, and conclude that $\Delta$ is independent of $\omega$. Using this $\Delta$ one can compute $\operatorname{deg}\left(\left(\pi_{\star} \omega_{C / S}\right)^{12}\right)=12 \operatorname{deg}\left(\pi_{\star} \omega_{C / S}\right)$ as in [40]. The result reads:

$h(C)=\frac{1}{12[K: Q]}\left(\log \left(\left|N_{K / Q}\left(\Delta_{C / K}\right)\right|\right)-\sum_{v \in s_{\infty}} \epsilon_{\nu} \log \left(\left|\Delta\left(\tau_{v}\right)\right|\left(i m \tau_{v}\right)^{6}\right)\right)$.

The standard modular form for $g=1$, of weight 12 called the discriminant is $2^{-4} \Delta$. This factor $2^{-4}$ is to make things work over $\mathbb{Z}$. There are also modular forms of weight 4,6 and so on. The reason to choose $\Delta$ is that it vanishes nowhere on the interior of the modulispace. This makes it possible to do the computation for all $C$ simultaneously.

The genus 2 case

Let $C$ be of genus 2. We will construct a canonical nonzero element $\Delta$ of $\left(\operatorname{det} H^{0}\left(C, \omega_{C / K}\right)\right)^{10}$. Its construction: let $\omega_{1}, \omega_{2}$ be a basis of $H^{0}\left(C, \omega_{C / K}\right)$, then $\omega_{1} / \omega_{2}$ is a rational function on $C$. This gives a morphism $C \underset{2: 1}{\rightarrow} \mathbb{P}_{K}^{1}$, let $R$ be the set of the 6 ramification points on $C$ of this map, then:

$$
\Delta:=\frac{\left.\prod_{P \neq Q}\left(\omega_{1} / \omega_{2}\right) P-\left(\omega_{1} / \omega_{2}\right) Q\right)}{\left(r e s\left(\omega_{1}^{2} / \omega_{2}\right)\right)^{20}} \cdot\left(\omega_{1} \wedge \omega_{2}\right)^{10}, \quad P, Q \in R
$$

where res $\left(\omega_{1}^{2} / \omega_{2}\right)$ is the residue of $\left(\omega_{1} / \omega_{2}\right) \omega_{1}$ at one of the two points where it has a pole (of order one). This $\Delta$ is independant of the choice of $\omega_{1}, \omega_{2}$. Using $\Delta$ one can compute $\operatorname{deg}\left(\left(\operatorname{det} \pi_{*} \omega_{C / S}\right)^{\otimes 10}\right)$ as in the genus one case. The result can already be found in [42], it reads:

$$
h(C)=\frac{1}{10[K: Q]}\left(\log \left(\left|N_{K / Q}\left(\Delta_{C / K}\right)\right|\right)-\sum_{v \in S_{\infty}} \epsilon_{v} \log \left(\left|\chi_{10}\left(\tau_{v}\right)\right|\left(\operatorname{detim} \tau_{\nu}\right)^{5}\right)\right) .
$$

*) This appendix was prepared in collaboration with B. Edixhoven. 
Note that as in the genus 1 case, our $\Delta$ differs from the standard one by a factor which is a power of 2.

For genus $\geqslant 3$ it is not possible to find a similar $\Delta$ which vanishes nowhere on the interior of the modulispace. For example, if $g=3$, one will have problems with the hyperelliptic locus.

Note added:

After this work was completed I received the CERN preprint TH 4660/87 Bosonization on higher genus Riemann surfaces, by L. Alvarez-Gaumé, J. Bost, G. Moore, P. Nelson and C. Vafa, in which aspects similar to those presented in section 2 have been discussed.

\section{ACKNOWLEDGEMENTS}

I am indebted to B. Edixhoven for explaining me various aspects of Faltings' work and for many useful comments. Also use of his results [19] in appendix $C$ is kindly appreciated. He also draw my attention to ref. [42] in which some of the results presented in section 4 were found independently. Furthermore I thank $\mathrm{H}$. Verlinde for inspirating discussions specifically on appendix B and showing me some explicit calculations [44]. I also thank M. Hazewinkel, B. de Wit and L. Alvarez-Gaumé for helpful discussions and reading the manuscript. This work is supported by "Stichting voor Fundamenteel Onderzoek der Materie".

\section{REFERENCES}

[1] L. AHLFors, Some remarks on Teichmüller's space of Riemann surfaces, Ann. Math. 74, 171, (1961).

[2] O. Alvarez, Conformal anomalies and the index theorem, Nucl. Phys. B216, 125, (1983), and in: Vertex operators in mathematics and physics, MSRI, Springer (1985).

[3] L. Alvarez-Gaumé, G. Moore, C. Vafa, Theta functions, Modular invariance and strings, Comm. Math. Phys. 106, 40, (1986).

L. Alvarez-GaumÉ and P. Nelson, Riemann surfaces and string theory, in Supersymmetry, Supergravity and Superstrings '86 (World Scientific, 1986).

[4] S. ARAKELOv, Intersection theory of divisors on an arithmetic surface, Izv. Akad. Nauk., 38, 1179, (1974).

[5] W. BAILY, On the embedding of $V$-manifolds in projective space, Am. J. Math. 79, 403, (1957).

[6] A. Beilunson, Yu. Manin, The Mumford form and Polyakov measure in string theory, Comm. Math. Phys. 107, 359, (1986).

[7] A. BeLAVIN, V. KNIZNIK, Algebraic geometry and the geometry of Quantum strings, Phys. Lett. 168B, 201, (1986). and: Complex geometry and theory of quantum strings Landau Institute preprint 1986-9.

[8] A. Belavin, V. Kniznik, A. Morozov and A. Perelomov, Two- and three-loop amplitudes in the bosonic string-theory, ITEP preprint (1986).

[9] A. Belavin, A. Polyakov, A.B. Zamolochikov, Infinite conformal symmetry in two dimensional quantum field theory, Nucl. Phys. B241, 333, (1984).

[10] L. Bers, Finite dimensional Teichmüller spaces and generalizations, Bull. Am. Math. Soc. (NS) 5, 131, (1981).

[11] J. BISMUT, D. FREED, The analysis of elliptic families: dirac operators eta invariants and the holonomy theorem of Witten, Comm. Math. Phys. 107, 103, (1986).

[12] J. Bost, Fibrés déterminants, déterminants régularisés et mesures sur les espaces de modules des courbes complexes, in Séminaire N. Bourbaki, no. 676, febr. (1987).

[13] J. BOST, T. JOLICOEUR: $A$ holomorphic property and the critical dimension in a string theory from an index theorem, Phys. Lett. B174, 273, (1986).

[14] R. Catenacci, M. Cornalba, M. Martellini and C. Reina, Algebraic geometry and path 
integrals for closed strings, Phys. Lett. 172B 328, (1986).

[15] T. CHINBERG, Introduction to Arakelov intersection theory, in Arithmetic Geometry eds. G. Cornell, J. Silverman, Springer (1986).

[16] P. Deligne, D. MumForD, The irreducibility of the space of curves of given genus, Publ. I.H.E.S. 36, 75, (1969).

[17] E. D'Hoker, D. Phong, Multiloop amplitudes for the bosonic Polyakov string, Nucl. Phys. B269, 205 (1986), and On determinants of Laplacians on Riemann surfaces, Comm. Math. 104, 537, (1986).

[18] R. ELKIK, Fonctions de Green, Volumes de Faltings Application aux surfaces Arithmétiques, Séminaire sure les pinceaux arithmétiques, Asterisque 127, ed. L. Szpiro (1985).

[19] B. EDIXHOVEN, private communication, and B. EDIXHOVEN, D.J. SMIT, in preparation.

[20] G. Faltings, Calculus on Arithmetic surfaces, Ann. Math. 119, 387 (1984).

[21] G. Faltings, G. Wü'stholtz et al., Rational points Seminar Bonn/Wuppertal 1983/84 Vieweg, Aspects of Mathematics, Vol. E6, Braunschweig-Wiesbaden, (1984).

[22] D. FreED, Determinants, Torsion and Strings, Comm. Math. Phys. 107, 483, (1986).

[23] D. FRIEDAN, Introduction to Polyakov's string theory, in: Les Houches 1983, North Holland 1984.

[24] D. Friedan, S. ShenKER, The analytic geometry of two dimensional conformal field theory, Nucl. Phys. B281, 509, 1987.

[25] C. GoMEZ, Modular invariance and compactification of the moduli space, Phys. Lett. 173B (1986).

[26] P. GRIFFITHS, J. HARRIs, Principles of algebraic geometry, p. 128 ff. Willey Interscience (1978).

[27] R. Gunning, Lectures on Riemann surfaces, Princeton University Press, Princeton NJ, (1966).

[28] J. HARRIS, D. MUMFoRd, On the Kodaira dimension of curves, Invent. Math. 67, 23, 1982.

[29] R. HARTSHORNE, Algebraic geometry, Springer (1977).

[30] N. KoBLITZ, p-adix numbers, p-adic analysis and zeta functions, Springer (1977).

[31] YU. MANin, Lectures on the K-functor in algebraic geometry, Russ. Math. Surv. 24 (5) 1, (1969).

[32] YU. MANIN, The partition function of the string can be expressed in terms of theta-functions, Phys. Lett. B172, 184 (1986).

[33] H. MASUR, The extension of the Weil Petersson metric to the boundary of Teichmuller space, Duke Math. J. 43, 623, (1979).

[34] G. Moore, P. Nelson, Measure for moduli, Nucl. Phys B266 58, (1986).

[35] L. Moret-Barluy, Metriques Permisses, Seminaire sur les princeaux arithmétiques, Asterisque 127, ed. L. Szpiro (1985).

[36] A. MoRozov, Explicit formulae for one, two, three and four loop string amplitudes, Phys. Lett. 184B, 177 (1987).

[37] D. MUMFoRD, On the stability of projective varieties L'Ens Math. 23, 39, (1977), and Towards an Enumerative Geometry of the Moduli space of curves, in Arithmetic and geometry, Birkhäuser 1983.

[38] A. Polyakov, Quantum geometry of bosonic string, Phy. Lett. 103B, 207, (1981), and Quantum geometry of fermionic string, Phy. Lett. 103B, 211, (1981).

[39] D. QuIllen, Determinants of Cauchy Riemann operators over a Riemann surface, Funkt. Anal. Prilozen. 19, 37, (1985).

[40] J. Silverman, The theory of height functions, and: Height and Elliptic curves, in Arithmetic geometry, eds. G. Cornell and J. Silverman, Springer (1986).

[41] J. TATE, Algorithm for determining the type of a singular fibre in an elliptic pencil in "Modular functions of one variable", IV, Lect. Notes. Math. 476 (Springer).

[42] K. UENO, Bosonic strings and arithmetic surfaces, Kyoto University preprint, 1987.

[43] E. VERLINDE, H. VERLINDE, Chiral bosonization, determinants and the string partition function, to appear in Nucl. Phys. B288, (1987).

[44] H. VERLINDE, private communication.

[45] P. VojtA, $A$ higher dimensional Mordell conjecture in Arithmetic Geometry, eds. G. Cornell, J. Silverman, Springer (1986).

[46] C. WOLPERT, On the Kählerform of the moduli space of once punctured tori, Comment. Math. Helv. 
58, 246, (1983).

[47] S. WolPERT, On the Homology of the moduli space of stable curves, Ann. Math. 118, 491, (1983).

[48] S. WOLPERT, Chern forms and the Riemann tensor for the moduli space of curves, Invent. Math. 85, 119, (1986). 
